\title{
Écriture d'invention et argumentation
}

Anne Vibert

\section{OpenEdition}

Journals

Édition électronique

URL : http://journals.openedition.org/recherchestravaux/339

DOI : 10.4000/recherchestravaux.339

ISSN : 1969-6434

\section{Éditeur}

UGA Éditions/Université Grenoble Alpes

\section{Édition imprimée}

Date de publication : 15 décembre 2008

Pagination : 35-87

ISBN : 978-2-84310-131-1

ISSN : 0151-1874

\section{Référence électronique}

Anne Vibert, «Écriture d'invention et argumentation », Recherches \& Travaux [En ligne], 73 | 2008, mis en ligne le 15 juin 2010, consulté le 07 septembre 2020. URL : http://journals.openedition.org/ recherchestravaux/339; DOI : https://doi.org/10.4000/recherchestravaux.339 
Anne VIBERT

Université Stendhal - Grenoble 3

Traverses I9-2 I (CEDILIT)

\section{Écriture d'invention et argumentation}

La majorité des sujets d'invention de l'Épreuve anticipée de français du baccalauréat (désormais EAF) demandent de produire un écrit de type argumentatif : le constat en a déjà été fait ${ }^{1}$. Or, on sait que la forme des sujets donnés au baccalauréat rétroagit fortement sur le type de sujets donné ensuite dans les classes et contribue ainsi à définir les pratiques scolaires dominantes de ce type d'écriture. Comment expliquer cette tendance forte, alors qu'on aurait pu attendre de l'introduction de l'écriture d'invention une ouverture vers d'autres formes d'écritures, les deux autres sujets (dissertation et commentaire) étant déjà des écrits de type argumentatif ? S'agit-il simplement d'une résistance à l'introduction de ces nouvelles formes d'écriture au lycée et d'une prégnance des genres scolaires de la dissertation et du commentaire?

Ce constat n'entend pas pour autant plaider pour une évacuation de l'argumentation de l'écriture d'invention. Mais la forme même de ces écrits argumentatifs dénote une absence de réflexion préalable sur la notion de genre argumentatif et une certaine méconnaissance théorique des enjeux de l'argumentation qui rendent difficile en amont tout apprentissage véritable de l'argumentation à travers des genres argumentatifs bien identifiés.

I. Les enseignants questionnés en 2005 par Fr. Le Goff notent le cloisonnement des écrits «dans une orientation argumentative» (Pratiques, n ${ }^{\circ}$ 27-I 28, décembre 2005, p. 69). Pour Y. Maubant également, dans sa contribution au même numéro de Pratiques ("Ambiguités de l'écriture d'invention au lycée : un champ non stabilisé») les difficultés de l'écriture d'invention «viennent du choix commun d'une écriture argumentative (ou à visée argumentative) et des genres prescrits par cette argumentation : lettre, essai, discours devant une assemblée, apologue...» (p. I73). Et V. Houdart-Mérot dans Réécriture et écriture d'invention au lycée, Hachette, 2004, trouve «très dommageable de réduire l'écriture d'invention au discours argumentatif déguisé» (p. 9). 
Il faut donc dans un premier temps préciser davantage ce qu'on entend par «sujet d'invention argumentatif » à travers l'analyse des sujets proposés depuis l'introduction de l'écriture d'invention dans les épreuves de français du baccalauréat, et essayer de mieux comprendre les raisons qui ont pu présider à un tel choix pour l'écriture d'invention.

Au-delà de l'écriture d'invention à l'EAF, le constat - sévère en termes didactiques - de l'absence de tout lien entre la forme argumentative du sujet d'invention et l'objet d'étude "Convaincre, persuader, délibérer» doit nous inviter à réfléchir sur la place de l'argumentation dans les programmes et à interroger le flou théorique qui y caractérise l'approche de l'argumentation.

Enfin, alors que la notion de genre oriente la quasi-totalité des consignes d'écriture d'invention, la question des genres argumentatifs n'est jamais clairement posée et, a fortiori, aucun enseignement-apprentissage de ces genres n'est proposé. C'est donc à cette absence qu'on proposera de remédier pour finir en suggérant un certain nombre de pistes didactiques².

\section{I. Écriture d'invention et argumentation : essai de taxinomie}

\section{A. Des sujets d'invention majoritairement argumentatifs}

\section{Taxinomie des sujets et comptage}

En l'absence d'une base de données officielle permettant d'avoir accès à l'ensemble des sujets proposés au baccalauréat depuis la dernière réforme, les sujets étudiés ont été consultés dans les annales constituées par le site Magister depuis $2002^{3}$. Ces annales offrent une base de 80 sujets. On peut donc considérer qu'elles donnent une idée assez exacte des tendances concernant les caractéristiques des sujets d'invention.

Sur les 80 sujets étudiés, 58 ont une forme argumentative clairement indiquée dans la consigne. Un certain nombre proposent ainsi une situation de controverse, de diptyque argumentatif, sous forme de dialogue ou d'échange de lettre, dans laquelle les points de vue à défendre sont indiqués comme opposés :

2. Cette contribution, qui dépasse la dimension d'un article standard, articule deux réflexions : l'une portant sur les sujets d'invention argumentatifs, l'autre sur l'argumentation dans les programmes du lycée. En fonction de ses intérêts et de son appétit, le lecteur pourra lire l'ensemble ou se contenter des parties i et 3 (les sujets d'invention argumentatifs et l'apprentissage des genres argumentatifs) ou simplement de la partie 2 ('’argumentation dans les programmes du lycée).

3. «Magister Travaux dirigés de français» : http://www.site-magister.com/, site consulté le 29 mai 2008. 


\section{ÉCRITURE D'INVENTION ET ARGUMENTATION}

Deux lecteurs débattent de leurs goûts en matière de poésie. Chacun défend un poème de ce corpus, l'un penchant pour les formes anciennes, l'autre pour les formes nouvelles. Vous rédigerez leur dialogue en veillant à y intégrer des exemples précis. (2003, toutes séries, sujet de secours)

Rédigez un dialogue argumentatif entre deux metteurs en scène ayant des conceptions opposées (interprétation des personnages, mise en scène) de la scène des Mains sales. (2004, série L, centre étrangers)

Un éditeur souhaite publier la correspondance intégrale d'un écrivain et tente de vaincre les réticences du descendant, qui est le seul à pouvoir lui donner l'autorisation de publication. Vous écrivez deux lettres, celle du descendant qui ne souhaite pas dévoiler toutes les facettes de l'écrivain, puis celle de l'éditeur, qui argumente pour le convaincre. (2004, série L, Amérique du Nord)

Michel Leiris défend le caractère poétique de son œuvre Langage, tangage... auprès de son éditeur qui refuse de publier le manuscrit dans sa collection «Poètes d'hier et d'aujourd'hui». Vous rédigerez la discussion qui les oppose, en vous référant également, si vous le jugez bon, à d'autres textes et à d'autres auteurs. (2006, série ES-S, centres étrangers)

D'autres sujets demandent une argumentation qui défend une position, indiquée par la consigne, mais sous une forme monologique. Le point de vue adverse n'est pas alors exprimé mais doit être pris en compte (soit par réfutation, soit par anticipation sur les objections possibles) :

En réponse à des critiques qui lui reprocheraient d'avoir engagé la littérature dans le combat politique, Victor Hugo écrit un article où il défend le choix de tout écrivain de mettre la littérature au service de grandes causes. (2002, série ES-S, centres étrangers)

Vous venez de retrouver dans un grenier les lettres que vos (arrière-)grandsparents avaient échangées jadis. Vous écrivez alors à un éditeur pour qu'il publie cette correspondance. Votre lettre fera preuve d'éloquence et devra présenter à votre destinataire des arguments et exemples en soulignant l'intérêt de cette publication tout en répondant par avance à ses objections. $(2003$, série $\mathrm{L}$, centres étrangers)

Imaginez la scène dans laquelle Macbeth, ou le père Ubu, ou Macbett, tente d'emporter l'adhésion d'un groupe de conjurés potentiels et répond à leurs objections. Vous respecterez le registre propre à la pièce choisie. (2004, série L, Guadeloupe-Guyane-Martinique)

Pour ces sujets, l'argumentation suppose un contre-discours, implicite ou exprimé dans le corpus, et le modèle d'argumentation reste donc clairement dialogal.

Enfin, une troisième catégorie de sujets ne se situe pas sur un terrain conflictuel : il ne s'agit pas d'argumenter pour modifier une opinion opposée ou a priori hostile, mais bien plutôt de faire partager un goût, une dilection, de justifier un choix de vie ou une position intellectuelle qui peuvent ne pas être compris par le ou les destinataires. Les termes de la consigne sont alors plus du côté de l'explication et de la clarification que de la controverse : 
Vous êtes chargé par votre professeur de français de constituer une anthologie qui rassemblera les poèmes que vous préférez. En préface à ce recueil, dans lequel figureront entre autres les poèmes du corpus, vous écrirez un texte qui présente vos choix et ce qui les a guidés. Vous aurez en particulier pour objectif de faire partager à vos camarades votre conviction que lire, ou éventuellement écrire, des poèmes peut apporter des remèdes aux maux de la vie. (2003, séries technologiques)

Dans un article pour un journal de lycéens, vous ferez à votre tour l'éloge d'un voyage accompli à pied ou par un autre moyen de déplacement, seul ou en groupe. Vous rédigerez un texte qui rende compte des sensations et émotions ressenties. (2006, séries technologiques, centres étrangers)

En vous inspirant des textes du corpus, vous écrirez les pages du journal qu'un jeune poète pourrait tenir lors de ses «escapades». Il y explique en particulier les raisons de ses fugues et son attrait pour l'écriture poétique. (2006, série ES-S, centres étrangers)

Comment comprendre une orientation aussi majoritairement argumentative dans les consignes de l'écriture d'invention? Trouvera-t-on dans les instructions officielles des préconisations qui expliquent ces choix des concepteurs des sujets?

\section{L'écriture d'invention dans les programmes: une définition ouverte}

On peut d'autant plus s'étonner de ce choix massif en faveur de l'argumentation que la définition de l'écriture d'invention dans les programmes ne semblait pas l'appeler. Le programme de seconde distingue trois types d'écrits que les élèves sont entraînés à produire : des écrits d'argumentation, des écrits d'invention et des écrits fonctionnels. Ces écrits d'invention sont en particulier «des imitations, des transformations et des transpositions des textes lus» et "permettent aux élèves de construire leur réflexion sur les genres et registres ${ }^{4} \gg$.

Dans le document d'Accompagnement des programmes, l'écriture d'invention se définit surtout par opposition à la dissertation et au commentaire, caractérisés l'une et l'autre comme «un texte qui en glose, commente, discute un autre», tandis que l'écriture d'invention consiste à «produire un texte nouveau, autonomes». Il est dit encore qu'elle est "production d'un texte, non sur un autre texte, mais à partir d'un ou plusieurs textes» (p. 90-9I), qu'«elle n'est pas a priori liée à un seul domaine, au seul narratif et à l'autobiographie ou à la fiction» mais qu'«elle peut porter sur tous les domaines et toutes les formes, le débat d'idées et le poétique aussi bien que le narratif» (p. 9I). Lors de la

4. Programmes. Français classe de seconde, CNDP, mai 2002, p. I6. Référence désormais abrégée : Progr. $2^{\text {de }}$.

5. Accompagnement des programmes de français. Français classes de seconde et de première, CNDP, septembre 200I, p. 89. Référence désormais abrégée : Acc. 
rédaction de l'Accompagnement des programmes, il s'agissait surtout de désamorcer la crainte d'une domination du narratif et d'une assimilation de l'écriture d'invention au type de sujets donnés au collège, d'où cette insistance sur une conception large des domaines et des formes. Mais rien n'est dit pour orienter l'écriture d'invention vers l'argumentation. En classe de première, la progression permet de réinvestir les domaines du narratif, «présent dans le biographique et - dans certains cas - l'épistolaire», du dialogue théâtral et du portrait «avec les récits de vie» et de la poésie en mettant à profit «les explorations faites en seconde», c'est-à-dire les jeux poétiques avec le langage. Mais il est dit aussi que «les genres étudiés en première peuvent y être les lieux privilégiés d'écriture d'invention : essai, dialogue, récit de vie, lettre le cas échéant» (Acc. p. 9I). Or, ces genres permettent une orientation argumentative, et ce sont eux qui ont eu tendance à s'imposer à l'examen.

Lorsqu'on se penche en effet sur les textes définissant l'écriture d'invention à l'épreuve anticipée du baccalauréat, on retrouve la différence marquée avec les deux autres types de sujet puisqu'elle "permet au candidat de mettre en œuvre d'autres formes d'écriture que celles de la dissertation ou du commentaire». Si l'on considère que dissertation et commentaire sont des genres argumentatifs, comme le précise d'ailleurs l'Accompagnement des programmes ${ }^{6}$, l'écriture d'invention devrait être au contraire un moyen de faire une large place à une écriture non argumentative. Mais l'énumération des genres inscrits au programme de première - article, lettre, monologue délibératif, dialogue, discours devant une assemblée, essai, récit à visée argumentative sous forme de fable, apologue - oriente l'écriture d'invention vers l'argumentation. Seule la série littéraire a droit à «amplification (écriture dans les marges ou les ellipses du texte), parodie et pastiche ${ }^{7} \gg$.

Pourtant, le lien avec les textes lus, qui caractérise l'écriture d'invention, ne devrait pas être limité à des genres argumentatifs : l'écriture d'invention en effet «contribue à une meilleure compréhension des textes lus parce qu'en imitant, transformant et transposant les modèles qu'ils y observent, les élèves se les approprient et peuvent être davantage sensibles aux particularités de style, de genre et de registres» (Acc. p. $92^{8}$ ). Très logiquement, on devrait

6. La dissertation est en effet «une formation à l'argumentation par des confrontations de points de vue» (Acc. p. 96) et le commentaire «est un exercice d'analyse et d'argumentation : il énonce des caractéristiques du texte et les prouve, par des références précises et des citations» (ibid. p. 97).

7. Note de service 200I-II 7 du 20-6-200I, Bulletin officiel, n² 26, 28 juin 200I. Pour les modifications mineures apportées à cette liste en 2006, voir infra ma troisième partie.

8. Voir également à propos des «Perspectives d'étude»: «La lecture des œuvres littéraires et l'écriture d'invention nourrissent la sensibilité, l'analyse et l'argumentation nourrissent le jugement» (Acc. p. I I). 
trouver des sujets d'invention argumentatifs principalement lorsque le corpus de texte concerne l'objet d'étude "Convaincre, persuader, délibérer». Mais ce n'est pas le cas puisque l'objet d'étude en question intervient 17 fois sur l'ensemble des sujets considéré et n'explique donc qu'un petit tiers des sujets argumentatifs répertoriés.

Pour expliquer ce lien constaté entre écriture d'invention et argumentation, on peut invoquer l'influence des modalités antérieures de l'EAF : l'analyse de textes argumentatifs dans les instructions officielles de I995, qui elle-même remplaçait le résumé-discussion'. On peut aussi montrer, comme le fait Bertrand Daunay ${ }^{10}$, à quel point, dans l'Accompagnement des programmes, le rapport n'est pas clair et les frontières sont floues entre écriture d'invention et argumentation puisqu'elles sont tantôt associées et tantôt dissociées. Une «Note sur la dissertation et l'écriture d'invention» destinée à la classe de première (Acc., p. 94) va même jusqu'à assimiler l'écriture d'invention et l'écriture argumentative ${ }^{\mathrm{II}}$. Mais les modalités de l'écriture d'invention sont suffisamment développées dans l'Accompagnement des programmes pour qu'on ne puisse douter de la volonté de rupture avec les exercices antérieurs et les explications évoquées ci-dessus ne sont pas suffisantes. S'il y a difficulté d'affranchissement, c'est sans doute beaucoup plus à l'égard des genres scolaires de la dissertation et du commentaire, comme un examen plus approfondi des sujets va permettre de le montrer.

\section{B. Le couplage entre forme argumentative et caractère métatextuel du sujet}

L'autre caractéristique frappante des sujets d'invention donnés à l'EAF depuis 2002 est leur caractère majoritairement métatextuel voire métapoétique. Alors qu'on attendrait un rapport de type hypertextuel avec les textes du

9. N. Denizot, dans «L'écriture d'invention au lycée : écriture des genres ou nouveau genre scolaire?» (Pratiques, n I 27-I 28, décembre 2005), montre que l'écriture d'invention est dans la droite ligne d'exercices qui avaient déjà cours avant 200I, lors des évaluations nationales en début de seconde, ou dans les séries techniques et technologiques, puisque le premier sujet de ces séries proposait déjà un écrit du type des écrits d’invention. Mais les sujets étaient cantonnés à l'argumentation et perçus comme une alternative au sujet de «discussion», non comme un type de sujet radicalement différent. La forme (discours, lettre) était secondaire par rapport à la visée argumentative, les compétences visées étant argumentatives et non génériques.

Io. Dans «Les liens entre écriture d'invention et écriture métatextuelle dans l'histoire de la discipline», article paru dans Enjeux, n 57 , septembre 2003, et dans Skholê, hors-série no I, 2004.

I I. Je ne reviendrai pas ici sur les raisons de ce brouillage, lié aux polémiques qui ont accompagné l'introduction de l'écriture d'invention au lycée. On se reportera pour cela à un autre article de B. Daunay, «Les ambiguïtés des textes officiels sur l'écriture d'invention», Pratiques, $\mathrm{n}^{\circ}$ I 27-I 28, décembre 2005, et dans le même numéro, à celui d'Y. Reuter, «L'écriture d'invention : réflexions didactiques sur une réforme en cours». 
corpus, en fonction des indications données par la définition de l'exercice ${ }^{12}$ et de la volonté, affichée par leurs auteurs, de rétablir un équilibre entre culture du commentaire et culture rhétorique ${ }^{13}, 44$ sujets sur 80 invitent les élèves à tenir un discours critique soit sur des textes, soit sur un genre, soit sur la littérature ou d'autres formes d'art, comme par exemple le sujet suivant qui demande une défense et illustration de la poésie :

Vous êtes directeur d'une revue poétique. À un lecteur ou une lectrice qui a affirmé que la poésie était inutile dans notre monde actuel, vous répondez sous la forme d'une lettre en prenant la défense de la poésie. Vous utiliserez les textes du corpus mais aussi les textes et les œuvres étudiés ou lus personnellement. Vous présenterez votre travail sous la forme d'une lettre mais sans la signer. (2007, série technologique, centres étrangers)

Bertrand Daunay a souligné que pourtant, dans les accompagnements de programme, l'écriture d'invention était opposée à l'écriture métatextuelle ${ }^{14}$, l'écriture d'invention étant "production d'un texte, non sur un autre texte mais à partir d'un ou de plusieurs textes» (Acc., p. 90). L'Accompagnement précise en outre : «La rédaction de commentaire et de dissertation appartient au domaine du discours critique sur la littérature» et rapproche l'écriture d'invention, qui constitue «un contact avec l'expérience littéraire de l'écriture», de l'expérience que les lycéens peuvent avoir des arts (dessin, peinture ou musique). Le texte officiel conclut ainsi le développement consacré aux objectifs de l'écriture d'invention :

[L]'étude de la littérature n'a pas pour seule fin de donner une attitude de critique : elle a pour fin de former le jugement et le goût pour la littérature et ses ressources propres, la sensibilité et l'exploration du langage; l'écriture d'invention a ce rôle. (Acc., p. 89)

L'orientation métatextuelle massive des sujets est donc en contradiction avec les objectifs de l'écriture d'invention tels que les définissent les textes officiels. Plus encore : 36 sujets sont non seulement métatextuels ou métapoétiques mais également argumentatifs. En voici quelques exemples :

Une revue littéraire prépare un numéro sur le récit autobiographique. Vous êtes chargé(e) d'y écrire un article dans lequel vous montrerez en quoi l'écriture autobiographique peut être une aide pour les autres et pour soi. Vous développerez votre argumentation en utilisant le texte du corpus et les lectures que vous avez faites par ailleurs. (2003, séries technologiques, centres étrangers)

I 2. «L'exercice [...] exige du candidat qu'il se soit approprié la spécificité des textes dont il dispose (langue, style, pensée) afin d'être capable de les reproduire, de les prolonger, de s'en démarquer ou de les critiquer» (Bulletin officiel, $\mathrm{n}^{\circ} 26$ du 28 juin 200 I).

I3. Voir A. Petitjean, A. Viala, «Les nouveaux programmes de français du lycée», Pratiques, n० 107-108, décembre 2000, p. 27.

I4. «Les ambiguïtés des textes officiels sur l'écriture d'invention», art. cité, p. 25. 
La comédie de L'Avare a été écrite et représentée en i 668. Il est question, dans la scène proposée, de costumes à la mode et d'autres qui sont démodés. Un comédien et son metteur en scène s'opposent sur le choix des costumes à faire porter aujourd'hui aux personnages : faut-il, pour donner à la scène tout son comique, garder les habits suggérés par le texte de Molière ou leur préférer des vêtements plus modernes? Vous rédigerez leur dialogue. (2004, série ES-S)

À sa parution, le texte de Hugo [= «Réponse à un acte d'accusation] suscite un vif débat dans la presse. Vous écrivez alors un article polémique, dans lequel vous défendez ou, au contraire, attaquez sa conception selon laquelle la poésie doit employer tous les moyens expressifs qu'elle désire. (2005, série ES-S)

Pour une revue de poésie créée avec des camarades, vous devez écrire un article intitulé : «Je n'aime pas les vers : j’aime la poésie!» Rédigez cet article en l'illustrant de citations tirées des textes du corpus et en vous aidant de vos lectures de classe ou personnelles. Vous donnerez à votre prose un ton convaincu, sans aucune familiarité. (2006, séries technologiques)

Comme l'a déjà constaté Nathalie Denizot après Bertrand Daunay, «bon nombre de sujets d'écriture d'invention ressemblent en fait à des dissertations, voire à des commentaires, brouillant la frontière de l'hyper et du métatextuel». Ce n'est donc pas, selon elle, «le rapport au texte-source (hypertextualité ou métatextualité) qui différencie écriture d'invention d'un côté, et commentaire ou dissertation de l'autre, mais bien le texte produit»: le sujet d'écriture d'invention «inscrit toujours sa production dans un genre écrit non scolaire, qu'il soit littéraire ou non littéraire» (art. cité, p. 35). Ces genres (lettre, article, dialogue, préface...) se prêtent en effet fort bien à l'écriture métatextuelle : nous y reviendrons.

Le brouillage entre écriture d'invention et dissertation est d'ailleurs explicitement instauré dans l'Accompagnement des programmes:

[...] la dissertation et l'écriture d'invention ne sont pas dans un rapport d'opposition ou d'exclusion mais de liaison et de complémentarité. La dissertation littéraire, quand elle porte sur un ensemble d'œuvres, se rapproche du débat d'idées qui peut donner matière à un essai, voire à un dialogue. D'autre part, la dissertation suppose une réflexion sur les idées et arguments à y exposer, donc un travail d'inventio : le cadre générique est donné, mais cette part de l'écriture d'invention est bien présente. On peut rappeler que, dans l'histoire de la dissertation, elle a été, au XVII ${ }^{e}$ et au XVIII ${ }^{e}$ siècle, une forme de débat d'idées et de critique littéraire, et que les mots d'essai et de dissertation ont été souvent, au moins à cette époque, regardés comme des quasi-synonymes. Aussi convient-il de faire percevoir aux élèves que l'une et l'autre forme d'écriture participent au même travail de construction d'une façon d'exprimer son point de vue, par une argumentation de conviction dans un cas, par une argumentation de persuasion ou une mise en images dans l'autre. (p. 94-95)

Ce rapprochement s'apparente à un tour de passe-passe qui joue sur les mots («invention» dans «écriture d'invention» ne peut être assimilée à l'inventio de la rhétorique comme nous le verrons plus loin) et fait comme si écriture 
d'invention et dissertation devaient viser le même objet : le débat d'idées et la critique littéraire, ignorant volontairement les autres possibilités d'écriture littéraire ouvertes par l'écriture d'invention. De fait, les sujets qui suivent s'apparentent à des dissertations dont la ligne argumentative aurait été fixée à l'élève :

À un ami qui vous écrit que la littérature n'a pas pour but d'apprendre à réfléchir mais de distraire et d'émouvoir, vous répondez que c'est elle plus que les autres arts qui donne une juste vue du monde. Votre lettre sera construite et s'appuiera sur des exemples précis empruntés aux textes du corpus et à votre expérience de lecteur. (2003, série L, centres étrangers)

Vous rédigerez la préface que pourrait écrire un auteur pour justifier la place centrale accordée à la mère dans son projet autobiographique. Votre texte ne comportera pas moins de deux pages. Vous vous appuierez sur votre lecture des textes du corpus. (2003, série L, centres étrangers)

Seule différence notable avec la dissertation : un destinataire fictif est indiqué, alors que ce destinataire - l'enseignant-correcteur et l'auditoire universel - reste implicite dans le cas de la dissertation ou du commentaire. Le plan de l'écrit d'invention demandé parait plus libre et donc moins contraignant que pour les genres scolaires, mais la nécessité d'organiser le propos fait partie des consignes implicites et, comme l'a montré Nathalie Denizot, c'est sur le modèle des genres scolaires que s'appuient les corrigés des annales (art. cité, p. 4I-42). Et si le modèle de la dissertation domine pour l'ensemble des exemples considérés, celui du commentaire est lui aussi très prégnant :

Après la lecture de ce corpus, un des poèmes vous parait correspondre plus particulièrement à ce que vous appréciez dans la poésie. Vous l'adressez à une revue de poésie et dans votre lettre d'accompagnement vous en défendez l'intérêt par rapport à d'autres types de poèmes. Vous rédigez cette lettre. (2002, série L)

En outre, pour certains sujets dont les consignes n'indiquent pas une orientation argumentative, le modèle sous-jacent semble bien être celui du commentaire :

À la réception de ce texte [«La Légende de l'homme à la cervelle d'or»], «la dame qui demande des histoires gaies» décide de répondre à Alphonse Daudet. Dans sa lettre, elle évoque les émotions et développe les réflexions que cette histoire lui a inspirées. (2006, série ES-S)

Un metteur en scène s'adresse à l'ensemble de son équipe (acteurs, scénographe, costumiers, éclairagistes...) pour définir ses choix d'interprétation de l'extrait d'Antigone (texte B) et donner ses consignes pour qu'elle devienne, lors du spectacle, une grande scène d'affrontement. Vous rédigerez son intervention. (2006, série L, Centres étrangers)

La demande de justification appuyée sur des exemples tirés du texte reste implicite mais c'est la connaissance du genre scolaire du commentaire qui permet à l'élève de comprendre ce qui est attendu dans l'exercice d'écriture. 
Certaines consignes dédoublent même la production écrite en demandant à la fois une production de type hypertextuel et le commentaire de cette production :

Vous participez à un concours d'écriture : dans un texte de quinze à vingt lignes de forme libre, vous célébrez un lieu réel ou fictif. Vous accompagnerez votre texte d'une courte présentation pour justifier vos choix d'écriture. (2005, série L, centres étrangers)

Certes, il est «difficile de voir dans l'écriture d'invention un seul et même genre scolaire» comme le souligne Nathalie Denizot: «entre la lettre, le discours, l'apologue ou le monologue délibératif, tout semble différer» (art. cité, p. 4I). Pourtant, quand le sujet demande par exemple de rédiger une lettre, celle-ci doit «répondre à un certain nombre d'attentes scolaires» (légitimité du destinataire, longueur du texte, registre de langue, contenus argumentatifs). Elle doit donc «à la fois ressembler à une vraie lettre (dans sa forme essentiellement) et en même temps ne pas être une vraie lettre». L'élève «doit avant tout montrer qu'il sait ou sait faire quelque chose, tout en faisant comme si la situation d'énonciation n'était pas fictive». Il s'agit - prescription paradoxale «d'écrire scolairement des genres non scolaires» (loc. cit.).

Dans les cas où une consigne d'orientation argumentative se combine avec une consigne d'écriture critique, c'est donc le modèle des genres argumentatifs scolaires, dissertation et commentaire, qui se devine à travers les genres non scolaires. Qu'en est-il alors dans les cas d'écriture argumentative où la finalité de l'argumentation n'est pas littéraire ou esthétique?

\section{Les sujets d'invention de type argumentatif non métatextuels}

Ces sujets entretiennent d'autres rapports avec les textes du corpus : il s'agit soit de les amplifier, de pratiquer une "greffe» (ajout d'une scène dans une pièce de théâtre, d'un dialogue dans un récit, continuation d'une lettre...) (Io cas), soit de les transposer en faisant varier le cadre temporel (4 cas) ou le genre (2 cas), soit encore d'imiter un genre (3 cas). Le rapport peut même être beaucoup plus lâche et tenir à un rapprochement thématique (6 cas) :

Vous venez de lire la lettre de Mme du Deffand. Vous écrivez à un ami ou une amie pour lui faire part de vos réactions face à cette lettre et pour lui présenter votre propre conception du bonheur. (2007, série L, centres étrangers)

Si les textes du corpus ont eux-mêmes un caractère argumentatif et concernent l'objet d'étude «Convaincre, persuader, délibérer», il y a alors une plus grande probabilité que le sujet d'invention soit argumentatif, ce qui est le cas pour I 5 sujets sur 22. Pour les 7 autres sujets, les objets d'étude concernés sont l'Épistolaire (2), le Biographique (2), les Réécritures (2) et le Théâtre (I). 


\section{ÉCRITURE D'INVENTION ET ARGUMENTATION}

Analysons plus précisément ces sujets. On peut en proposer le classement suivant qui distingue ce que j'appellerai des «genres non marqués» dont les contraintes formelles sont faibles, et les genres ou registres littéraires aux contraintes formelles beaucoup plus importantes. Si dans les deux cas la situation d'énonciation est fictive, dans le premier cas, comme nous l'avons vu plus haut, le modèle implicite est en réalité celui des genres scolaires tandis que dans le second, les modèles sont littéraires. On peut cependant distinguer dans les «genres non marqués» (pour l'essentiel, la lettre, l'article et le

\begin{tabular}{|c|c|c|}
\hline $\begin{array}{l}\text { Genres non marqués } \\
\text { (situation d'énonciation } \\
\text { fictive mais peu éloignée } \\
\text { des genres scolaires) }\end{array}$ & $\begin{array}{l}\text { Genres non marqués mais } \\
\text { s'inscrivant dans une œuvre } \\
\text { littéraire }\end{array}$ & $\begin{array}{l}\text { Genres ou registres } \\
\text { littéraires avec contraintes } \\
\text { formelles fortes (situation } \\
\text { d'énonciation fictive } \\
\text { éloignée des genres } \\
\text { scolaires) }\end{array}$ \\
\hline $\begin{array}{l}\text { - lettre de G. Sand à } \\
\text { Baudelaire (condition des } \\
\text { femmes artistes) (2002) Biogr. } \\
\text { - discours de l'élève en tant } \\
\text { que délégué au comité de vie } \\
\text { lycéenne de l'établissement à } \\
\text { ses camarades pour convaincre } \\
\text { de la nécessaire participation } \\
\text { de la jeunesse au devoir de } \\
\text { mémoire (2004) Arg. }{ }^{15} \\
\text { - lettre de Lily à sa famille en } \\
\text { Somalie dénonçant intolérance } \\
\text { et racisme (2005) Arg. } \\
\text { - dialogue entre deux } \\
\text { interlocuteurs défendant } \\
\text { chacun leur conception du } \\
\text { bonheur (2005) Arg. } \\
\text { - lettre de l'élève au courrier } \\
\text { des lecteurs d'un journal pour } \\
\text { dénoncer une injustice }+ \\
\text { énoncé d'une morale (2005) } \\
\text { Arg. } \\
\text { - article de l'élève pour un } \\
\text { journal de lycéens (éloge d'un } \\
\text { voyage) (2006) Biogr. } \\
\text { - lettre de l'élève à un élu } \\
\text { local pour raconter une scène } \\
\text { proche de celle des «Effarés» } \\
\text { et l'inciter à agir ( } 2007 \text { ) Arg. } \\
\text { - lettre de l'élève à un ou une } \\
\text { amie pour réagir à la lettre de } \\
\text { Mme du Deffand et présenter } \\
\text { sa propre conception du } \\
\text { bonheur (20o7) Épist. }\end{array}$ & $\begin{array}{l}\text { - suite de la lettre de la mère } \\
\text { d'Angelo développant la thèse } \\
\text { paradoxale soutenue par le } \\
\text { personnage («il faut être fou») } \\
\text { (2002) } \\
\text { Épist.- dialogue entre le roi } \\
\text { Roger et le pèlerin sur la } \\
\text { conception du bonheur (2004) } \\
\text { Arg. } \\
\text { - dialogue entre LuI et MoI à } \\
\text { partir de deux répliques sur un } \\
\text { même thème (amplification } \\
\text { et possibilité de transposition } \\
\text { contemporaine) (2006) Arg. } \\
\text { - lettre du roi à Marot en } \\
\text { réponse à sa requête (se } \\
\text { considère comme injustement } \\
\text { emprisonné) (2006) Arg. } \\
\text { - lettre du sultan Yaya à } \\
\text { Osman tirant la leçon de } \\
\text { l'épisode de la gazelle (2007) } \\
\text { Arg. }\end{array}$ & $\begin{array}{l}\text { - tirade théâtrale d'Hector sur } \\
\text { le thème de la guerre (2002) } \\
\text { Arg. } \\
\text { - récit en prose sur le pouvoir } \\
\text { avec moralité (2003) Arg. } \\
\text { - dialogue théâtral Ismène- } \\
\text { Antigone (défense des lois de } \\
\text { la cité) (2003) Réécr. } \\
\text { - plaidoyer en faveur de } \\
\text { l'humanité (réponse au } \\
\text { réquisitoire du juge) (2004) } \\
\text { Arg. } \\
\text { - narration à valeur } \\
\text { d'apologue (dans une lettre) } \\
\text { pour dissuader un proche de } \\
\text { commettre une erreur grave } \\
\text { (2004) Arg. } \\
\text { - scène dans laquelle } \\
\text { Macbeth, père Ubu ou } \\
\text { Macbett tente d'emporter } \\
\text { l'adhésion d'un groupe de } \\
\text { conjurés (respect du registre) } \\
\text { (2004) Réécr. } \\
\text { - monologue délibératif où } \\
\text { Béranger doit choisir entre ses } \\
\text { sentiments et ses habitudes } \\
\text { (2004) Théâtre } \\
\text { - apologue dont le héros } \\
\text { symbolise le xxI siècle } \\
\text { commençant (2005) Arg. } \\
\text { - discours de V. Hugo à la } \\
\text { Chambre des pairs pour plus } \\
\text { de justice sociale (2007) Arg. }\end{array}$ \\
\hline
\end{tabular}

I 5. Arg. = objet d'étude « Convaincre, persuader, délibérer». 
dialogue), ceux qui s'inscrivent dans une ouvre littéraire sous la forme d'une greffe ou d'une amplification: la contrainte est en principe plus forte, puisqu'il s'agit de reprendre à son compte la parole de personnages de fiction et d'imiter également le style de l'écrivain. Mais la formulation des consignes permet de penser que l'accent est mis davantage sur les idées à développer que sur l'imitation littéraire et il y a fort à parier que c'est le modèle de la dissertation qui risque également de s'imposer pour ces sujets.

On constate, à la lecture du tableau de la page précédente, que ce sont les genres «non marqués» qui dominent. Alors qu'on pourrait attendre une correspondance entre les genres argumentatifs des textes du corpus, pour les I 5 sujets concernant l'objet d'étude "Convaincre, persuader, délibérer», et la forme argumentative du sujet d'invention, dans 5 cas, l'écrit à produire relève d'un autre genre que celui des textes du corpus : une lettre à partir d'une chanson engagée; un dialogue alors que les textes du corpus sont un apologue, une pensée et une rêverie; une lettre de dénonciation alors que le corpus propose des apologues ${ }^{16}$; un discours alors que le texte source est un témoignage; une lettre à partir d'un poème. Dans ces cas, les textes du corpus ne peuvent servir de modèle aux élèves pour la forme de leur écrit d'invention.

Plus que par la forme ou le genre argumentatifs, on peut caractériser ces sujets par leur enjeu, moral ou axiologique. Puisqu'il ne s'agit pas ici de traiter d'une question littéraire comme le proposent la majorité des sujets d'invention, il faut trouver une autre visée à l'argumentation : révolte contre l'injustice, dénonciation de l'intolérance, devoir de mémoire, guerre ou paix, pouvoir et obéissance aux lois pour les sujets à enjeu "collectif »; choix de vie, conception du bonheur, pour les sujets qui font appel à des choix personnels. On pourrait donc penser qu'on est là au cœur des enjeux de l'argumentation, mais entre les situations de communication factices (discours de l'élève en tant que délégué au comité de vie lycéenne de l'établissement à ses camarades pour convaincre de la nécessaire participation de la jeunesse au devoir de mémoire, lettre de l'élève au courrier des lecteurs d'un journal pour dénoncer une injustice), les bons sentiments comme thème obligé (dénonciation de l'intolérance ou de l'injustice), et les considérations sur le bonheur, on perd de vue la visée persuasive de l'argumentation et on voit se profiler la dissertation comme modèle d'écriture. Les situations de théâtre, parce qu'elles proposent une situation de communication fictive mais dont l'élève peut comprendre l'enjeu pour les personnages, permettent davantage l'exercice d'une argumentation en situation qui ne se réfère pas aux genres scolaires (par exemple, écrire la scène dans laquelle Macbeth, père Ubu ou Macbett tente d'emporter l'adhésion d'un groupe de conjurés).

I6. La consigne demande néanmoins que la lettre conduise à l'énoncé d'une morale! On garde la morale de l'apologue sans sa forme narrative, ce qui n'a guère de sens. 
Au regard de cette taxinomie, il apparait que l'écriture d'invention a beaucoup de mal à s'émanciper des genres scolaires de la dissertation et du commentaire lorsqu'il s'agit d'argumentation. Pour mieux comprendre cette situation, il est nécessaire d'examiner de plus près la manière dont les programmes et les textes d'accompagnement abordent l'argumentation.

\section{L'argumentation dans les programmes du lycée}

On ne saurait refaire ici l'histoire du retour ou de l'introduction ${ }^{17}$ de l'argumentation dans les programmes. Je renverrai pour cela à un article d'Alain Boissinot qui revient sur cette "émergence de l'argumentation au cœur de la réflexion didactique» à partir des années $1980^{18}$. Et je n'entends pas non plus remettre en cause le choix fait par les programmes de former les élèves à l'argumentation : bien au contraire, c'est au nom de la nécessité de cette formation que j'entends mener la critique qui suit. Alain Boissinot lui-même, qui fut pourtant un des premiers à proposer la transposition didactique des différentes théories de l'argumentation ${ }^{19}$, écrivait en 1999 à propos du nouveau programme de la classe de troisième et du programme en préparation de la classe de seconde :

Je ne vais pas me plaindre de cet engouement pour l'argumentation même si, comme toujours dans ces cas-là, on a un peu le sentiment que le balancier part tellement loin dans le sens où on avait tenté de donner une impulsion qu'on est préoccupé par les excès que cela peut engendrer. (art. cité, p. I)

Et il évoquait des problèmes de rigueur dans la définition de l'argumentation et la question de son articulation avec les textes littéraires. Si la critique des programmes du lycée est aujourd'hui possible, c'est parce qu'ils ont tenté la difficile transposition didactique d'un champ théorique non unifié, issu de disciplines différentes. Après sept années d'application de ces programmes, on peut tenter non de repartir en sens inverse, comme on a trop souvent coutume de le faire dans l'Éducation nationale ${ }^{20}$, mais tout au contraire de

17. Le choix du terme dépend de la façon dont on pense le lien de l'argumentation avec la rhétorique, soit comme une façon de revenir à la discipline qui prenait en charge l'enseignement de l'argumentation, soit comme une approche nouvelle issue d'autres disciplines et qui prend ses distances avec l'ancienne rhétorique.

I 8. "Argumentation et littérature», novembre i 999. Publié le $\mathrm{I}^{\text {er }}$ septembre 2000 par la Direction générale de l'enseignement scolaire sur Eduscol, «Formation continue des enseignants, Publications». Disponible sur: http://eduscol.education.fr/Do033/FXNRENo6. htm, consulté le 17 mai 2008.

I9. Dans son ouvrage Les textes argumentatifs, Toulouse, Bertrand-Lacoste / CRDP MidiPyrénées, I992, réédité en 1996.

20. Et le projet de programme pour le collège, soumis à la consultation au moment de la rédaction de ces lignes, en est un bon exemple: on est passé d'un programme organisé 
clarifier les choix théoriques pour améliorer l'enseignement de l'argumentation en général et sa pratique dans l'écriture d'invention en particulier.

\section{A. Une difficile transposition didactique}

Un premier constat s'impose : si l'argumentation est désormais très présente dans les programmes, elle y reste un objet aux contours imprécis. La transposition didactique des différentes approches de l'argumentation relève parfois plus d'un bricolage que d'une véritable construction et d'une articulation réfléchie ${ }^{21}$.

\section{La «nowvelle rhétorique» de Perelman}

Alors qu'on aurait pu attendre sinon un retour à la rhétorique qui, la première dans notre tradition scolaire, a permis de penser l'argumentation, du moins une prise en compte de ce que son héritage, récemment redécouvert dans son intégralité, peut apporter tant à l'analyse des textes qu'à la production écrite, les programmes ont très peu sollicité l'approche rhétorique de l'argumentation : j'y reviendrai plus loin pour m'interroger sur les raisons de cet «oubli».

C’est en revanche la «nouvelle rhétorique», celle de Chaïm Perelman, qui semble avoir inspiré les auteurs des programmes. Perelman est d'ailleurs la seule référence savante explicite des textes officiels puisque l'accompagnement du programme de troisième cite, dans sa partie intitulée «Éléments

à partir des formes de discours à un programme qui met au premier plan la grammaire de phrase; alors que l'argumentation occupait pratiquement la moitié du champ des discours, la notion de discours argumentatif disparaît et l'argumentation est désormais réduite à la portion congrue : l'initiation à la rédaction de paragraphes argumentés en $4^{\mathrm{c}}$ (pour répondre à des questions de lecture analytique), la présentation d'une prise de position étayée par quelques arguments et exemples en $3^{\mathrm{e}}$, l'apprentissage du type d'écrit argumentatif étant renvoyé au lycée. Le programme précise encore pour la $3^{\mathrm{e}}$ : «Le professeur apprend à l'élève à combiner expression de soi, récit et argumentation. Il privilégie également la rédaction de réponses argumentées à des questions portant sur les textes littéraires, notamment à l'aide d'un lexique approprié et de références claires aux passages étudiés. Le programme de Troisième invite l'élève à s'interroger sur les problèmes de l'humanité et les grandes questions de notre monde et de notre temps. Le professeur propose donc régulièrement à l'élève des travaux écrits l'incitant à donner son avis en le justifiant.»

2I. Dans les limites de cette contribution, je ne peux revenir sur les différentes théories de l'argumentation. Je me permets de renvoyer, pour le rappel des théories de référence transposées dans les programmes, à l'article de Cl. Garcia-Debanc, «L'enseignement de l'argumentation au lycée: entre approche linguistique et héritage rhétorique», Pratiques, n ${ }^{\circ}$ I I-I I 2 , décembre 200I, p. I 89-197. On peut renvoyer également à l'ouvrage précurseur d'A. Boissinot, déjà cité, même s'il est antérieur aux programmes du lycée, dans la mesure où il les inspire. Pour les théories elles-mêmes et la question de la confrontation des différentes approches issues de disciplines différentes, voir notamment L'Argumentation aujourd'bui. Positions théoriques en confrontation, textes réunis par M. Doury et S. Moirand, Presses Sorbonne Nouvelle, 2004. 
pour une approche de l'argumentation ${ }^{22} »$, une définition de l'argumentation avec sa référence à L'Empire rhétorique. Rhétorique et argumentation (Vrin, I977, p. 23). Si les programmes du lycée ne citent pas directement Perelman, la définition de l'argumentation qu'ils retiennent comme «forme de discours qui vise à obtenir l'adhésion d'un auditeur ou d'un lecteur aux thèses qu'on présente à son assentiment» (Acc., p. 2I) est très proche de la citation de Perelman retenue dans l'accompagnement du programme de troisième («provoquer ou accroître l'adhésion d'un auditoire aux thèses qu'on présente à son assentiment»). À Perelman encore est reprise la distinction entre les trois verbes qui définissent l'objet d'étude de la classe de seconde : «Démontrer, persuader, convaincre». La distinction entre «démontrer» et «argumenter» est à la base même du système de Perelman puisque son entreprise est tout entière conçue pour s'opposer à la réduction du raisonnement à la démonstration sur le modèle mathématique, selon l'idéal cartésien du raisonnement more geometrico. Il s'agit pour lui de montrer au contraire que la raison est compétente même dans les domaines qui échappent au calcul, d'où une théorie de l'argumentation qui s'intéresse non à la vérité des propositions, mais à l'adhésion des esprits au raisonnement qu'on leur propose. Cependant, en reprenant telle quelle cette dichotomie ${ }^{23}$, les programmes ne savent au fond que faire de la démonstration qui «se déroule hors de tout autre contexte que celui de son propre système» (Acc., p. 4I) et se contentent de suggérer :

Il peut être à cet égard utile d'aborder des textes scientifiques de haute qualité d'écriture (de Poincaré, Monod, Rostand), pour éclairer cette logique, comme une initiation à reprendre en première. (loc. cit.)

Non seulement la référence est très vague (quels textes?), mais il n'est pas sûr que ces «textes scientifiques de haute qualité d'écriture» relèvent de la démonstration, quand bien même ils seraient écrits par des scientifiques. En l'occurrence, la reprise des catégories de Perelman ne permet guère d'aborder le discours scientifique puisque, comme le remarque Christian Plantin, l'antagonisme argumentation/démonstration qui caractérise l'approche de

22. Enseigner au collège. Français. Programmes et accompagnements, CNDP, i999, p. I69. Référence désormais abrégée : Coll.

23. Chr. Plantin la commente ainsi : «Si l'on voulait analyser cette stratégie dans les termes mêmes du Traité, on dirait que le couple argumentation/démonstration est un "couple antagoniste", dont les termes font l'objet d'une véritable "rupture de liaison" ou "dissociation" (TA, 550). Systématiquement, on n'y parle de démonstration que comme repoussoir de l'argumentation, comme on peut le vérifier sur chaque occurrence du terme démonstration mentionnée à l'index. Cette stratégie de l'épouvantail constitue une des cellules génératrices fondamentales du Traité», L'argumentation, PUF («Que sais-je?»), 2005, p. 76. 
Perelman «se concrétise par l'exclusion du Traité de tout ce qui touche aux sciences $\left.{ }^{24}\right)$.

Quant à la distinction entre «convaincre» et «persuader», Perelman n'en est pas l'inventeur mais il la reprend à son compte en l'appuyant sur la notion d'auditoire. Le développement sur «Persuader et convaincre» suit en effet celui qu'il consacre à l'«Adaptation de l'orateur à l'auditoire ${ }^{25} »$. Bien qu'il se réfère à Pascal, notamment pour opposer à la conviction, qui s'adresse à la raison, la persuasion qui parle au corps, à l'imagination, au sentiment ${ }^{26}$, il conteste ce critère de distinction et en propose un autre, entendant ainsi se rapprocher plutôt de la conception kantienne :

Nous nous proposons d'appeler persuasive une argumentation qui ne prétend valoir que pour un auditoire particulier et d'appeler convaincante celle qui est censée obtenir l'adhésion de tout être de raison. (TA, p. $\left.{ }_{3} 6\right)$

La distinction entre persuader et convaincre permet ensuite à Perelman d'en venir à la notion d'auditoire universel, auditoire visé par celui qui cherche à convaincre et notamment par le philosophe.

Il ne s'agit pas ici de discuter les choix de Perelman mais de montrer que cette distinction entre «convaincre» et «persuader» est loin d'aller de soi et d'être inscrite dans la langue : elle fait l'objet d'une construction dans un système philosophique et n'est pas forcément admise comme vérité en dehors de ce système. Or, que font les programmes et accompagnements avec ces notions? L'objet d'étude «Démontrer, convaincre et persuader» est ainsi défini dans le programme de seconde:

Le but est de percevoir et comprendre les différences, mais aussi les liens, entre démontrer - dans le domaine des vérités vérifiables - et convaincre ou persuader, en s'appuyant sur des arguments rationnels ou sur des facteurs affectifs. (Progr. $2^{d e}$, p. I4)

Le programme ne retient ici que l'opposition conviction-raison/persuasionaffects que prétendait justement remettre en cause Perelman. L'Accompagnement des programmes tente de ménager à la fois cette opposition et la distinction perelmanienne :

24. Ibid., p. 77 .

25. Dans la première partie du Traité: «Les cadres de l'argumentation» (Ch. Perelman, L. Olbrechts-Tyteca, Traité de l'argumentation [1958], Éditions de l'université de Bruxelles, $5^{\mathrm{e}}$ édition, 2000, désormais $T A$ ).

26. Traité, op. cit., p. 37. On ne trouve pas cependant chez Pascal l'opposition entre convaincre et persuader. On la trouve en revanche formulée par d'Alembert dans ses Synonymes: «La conviction tient plus à l'esprit, la persuasion au cœur; ainsi on dit : l'orateur doit non seulement convaincre, c'est-à-dire prouver ce qu'il avance, mais encore persuader, c'est-à-dire, toucher et émouvoir», Euvres, t. 4, Martin Bossange et Cie, I 822, p. 255. 


\section{ÉCRITURE D’INVENTION ET ARGUMENTATION}

Celui qui cherche à convaincre s'attache au cheminement des raisons qui conduiront au résultat espéré : l'adhésion réfléchie de l'auditoire. [...] Même s’il ne s'adresse qu'à un seul interlocuteur, celui qui cherche à convaincre vise à travers celui-ci un destinataire plus général. L'adhésion ne s'obtient que dans la mesure où l'interlocuteur individuel peut reconnaître son appartenance à un auditoire général ou même universel. Les démarches de la conviction relèvent de la raison, du dialogue et de l'échange; elles sollicitent le savoir. [...]

Celui qui veut persuader cherche à obtenir une adhésion spontanée et affective de son destinataire. La persuasion vise un destinataire particulier - individuel ou collectif - dont on sollicite les attentes, les rêves ou les émotions [...]. La persuasion relève davantage que la conviction de la suggestion, de la séduction, de la tentation; elle sollicite moins le savoir que le désir ou la crainte. (Acc., p. 4I)

On voit dans ce passage comment le glissement s'opère d'une conception perelmanienne de la conviction (puisqu'il s'agit pour convaincre de viser «un auditoire général ou même universel») à la seule opposition raison/émotions.

Ces emprunts à Perelman posent un certain nombre de problèmes. Tout d'abord, il s'agit d'emprunts qui ne s'avouent pas comme tels et présentent comme un discours de vérité ce qui est la construction théorique d'un auteur. Ensuite, cet auteur n'a pas élaboré sa pensée théorique pour contribuer aux études littéraires. Perelman était un philosophe du droit dont l'objectif n'était ni l'étude des textes littéraires, ni la production d'écrits d'invention mais l'élaboration d'une logique non formelle en matière sociale, politique et philosophique ${ }^{27}$. Enfin, si les auteurs des programmes ont repris certaines définitions à Perelman, ils ne se sont inspirés que de sa réflexion sur les cadres de l'argumentation et ont complètement laissé de côté ce qui fait l'essentiel du Traité de l'argumentation, à savoir la réflexion sur les lieux et les données de départ de l'argumentation et l'étude des techniques argumentatives. Ainsi, on ne retrouve pas dans les programmes le classement, même simplifié, des types d'arguments proposé par Chaïm Perelman et Lucie Olbrechts-Tyteca. Il est simplement préconisé en seconde d'aborder «les grandes formes de raisonnement» : raisonnement par déduction, raisonnement inductif, raisonnement critique et raisonnement par concession (Acc., p. 22), catégories très générales qui ne constituent pas une description rigoureuse des modes de raisonnement et ne peuvent prétendre rendre compte de l'étude des «moyens discursifs d'obtenir l'adhésion des esprits» (TA, p. Io) qui était l'ambition des auteurs du Traité de l'argumentation.

27. «Les logiciens se doivent de compléter la théorie de la démonstration [...] par une théorie de l'argumentation. Nous chercherons à la construire en analysant les moyens de preuve dont se servent les sciences humaines, le droit et la philosophie; nous examinerons des argumentations présentées par des publicistes dans leurs discours, par des avocats dans leurs plaidoiries, par des juges dans leurs attendus, par des philosophes dans leurs traités", Traité de l'argumentation, op. cit., p. I3. 


\section{Approches linguistiques de l'argumentation}

L'approche de l'argumentation dans les programmes doit aussi beaucoup à la linguistique de l'énonciation et à la pragmatique. Avant même d'être un «objet d'étude», l'argumentation figure dans les programmes au titre d'une des «perspectives d'étude» qui doivent «amener les élèves à savoir construire les significations des textes et des œuvres» (Progr $2^{d e}$, p. I3). Conçue comme une démarche intellectuelle, la "perspective argumentative (ou pragmatique) doit «rendre compte du fait que les textes ne sont pas écrits et lus dans un monde idéal, mais justement dans [un] jeu de rapports humains» (Acc., p. I I). Il s'agit notamment de mettre en œuvre «l'étude de l'argumentation et les effets de chaque discours sur ses destinataires ${ }^{28} »$. L'emploi même du mot «discours» marque l'inscription dans une démarche pragmatique. Le discours est d'ailleurs défini, dans la partie de l'Accompagnement consacrée à l'étude raisonnée de la langue, comme toute «mise en pratique de la langue», elle-même «définie à partir de trois critères : celui de l'énonciation, celui de l'interaction et celui de l'usage» :

Cette définition fonde dans ses grandes lignes le programme de français au collège où on entend le discours comme une activité de langage individuelle réalisée en situation (énonciation), déterminée par des codes (usages) et comportant une visée (interaction). Les grandes perspectives d'étude qui structurent la conception des programmes de français au lycée trouvent là un point d'appui essentiel. (Acc., p. 7I)

Dans le développement consacré à l'interaction, l'Accompagnement des programmes précise qu'elle est « essentielle dans l'étude de l'argumentation, qui consiste à influencer autrui et à modifier son opinion» (p. 7I). Toujours dans la partie consacrée au discours, la modalisation, définie comme «ce qui module ou modifie la relation du sujet avec son énoncé», et qualifiée d'«essentielle dans l'étude ou la pratique de l'argumentation», fait l'objet de plusieurs développements (Acc., p. 72-74). Mais curieusement, alors que les textes officiels semblent vouloir se référer à la linguistique de l'énonciation et à la pragmatique pour l'étude du discours, leur présentation des connecteurs parait ignorer ces théories de référence, énumérant sur le même plan «mais», «au contraire», «en revanche», «tandis que» pour l'expression de l'opposition ou «car», «en raison de», «parce que», «puisque» pour celle de la cause. Il est simplement dit: «Ces divers éléments de l'organisation textuelle doivent être $[\ldots]$ mis en

28. Programmes. Français, classe de $I^{\text {re }}$ séries générales et technologiques. Littérature classe terminale série littéraire, CNDP, 2002, p. I 2 (référence désormais abrégée Progr. $I^{r}$ ). Rappel des autres perspectives : approche de l'histoire littéraire et culturelle; étude des genres et des registres; réflexion sur la production et la singularité des textes. Ou encore : perspective historique, générique, intertextuelle. 
relation avec la dimension du discours, c'est-à-dire avec l'énonciation» (Acc., p. 70). On peut certes penser que les rédacteurs du document d'accompagnement ont reculé devant la difficulté de transposition didactique des théories d'Anscombre et Ducrot sur L'Argumentation dans la langue ${ }^{29}$, mais alors pourquoi ces développements sophistiqués sur la modalisation et l'importance qui lui est accordée en rapport avec l'argumentation? Il y a là des choix dont la cohérence nous échappe.

Puisque «tout texte tient à une situation, relève d'un genre, tend à produire un effet sur ses lecteurs ou auditeurs, fait écho à d'autres textes» (Acc. p. II), tous les textes pourront être abordés du point de vue de leur enjeu argumentatif, même si, rappelle l'Accompagnement, il faut hiérarchiser les perspectives: "Laisser croire, par exemple, que la poésie n'a pas d'enjeu argumentatif et l'essai pas d'enjeu esthétique serait faux, mais oublier que l'enjeu esthétique peut être essentiel pour l'une, et le rôle du débat d'idées pour l'autre, ne le serait pas moins en retour» (ibid.). Reste néanmoins que l'articulation n'est pas vraiment faite avec les objets d'étude «Démontrer, convaincre, persuader» et «Convaincre, persuader, délibérer» dont l'approche est empruntée à la «nouvelle rhétorique» et non à la linguistique. Si tout texte peut être abordé dans une perspective argumentative, pourquoi des objets d'étude consacrés à l'argumentation? Y aurait-il dans les programmes une réflexion sur les genres de l'argumentation?

\section{Entre linguistique textuelle et approche générique}

Une didactique de l'argumentation ne peut manquer de s'appuyer sur la référence à des formes entérinées et répertoriées, susceptibles de déterminer un horizon d'attente pour la lecture comme pour la parole et l'écriture. Mais les programmes et accompagnements mêlent des approches d'origine théorique différente qui, dans ce cas encore, ne sont pas articulées.

Les programmes du lycée font en effet référence aux «formes de discours». Elles sont énumérées dans la liste de notions grammaticales de l'Accompagnement des programmes du lycée : discours argumentatif (démontrer, convaincre, persuader, délibérer), descriptif, narratif (narration, narrateur), explicatif (p. 74). Alors que ces formes de discours ne figurent ici qu'à titre de notions linguistiques, elles sont au principe même de la progression et des orientations générales des programmes du collège dont la finalité est de «donner aux élèves la maitrise des principales formes de discours» (Coll., p. 65). Ces formes sont réparties «selon deux pôles : le pôle narratif et le pôle argumentatif », le premier englobant le discours narratif et le discours

29. J.-Cl. Anscombre et O. Ducrot, L'Argumentation dans la langue, Liège-Bruxelles, Mardaga, I 980. 
descriptif, le second le discours argumentatif et le discours explicatif, répartition contestable que les accompagnements de programme sont d'ailleurs obligés de remettre en cause ${ }^{30}$, et leur étude s'organise selon une complexification progressive et non linéaire qui va de la narration à l'argumentation en passant par la description et l'explication, chaque forme de discours étant étudiée prioritairement à un niveau donné mais découverte au préalable au cours des années ou de l'année antérieure ${ }^{31}$.

L'origine de cette distinction de «formes de discours» doit être recherchée du côté des «types de textes» et donc de la linguistique textuelle. Si JeanMichel Adam, un des principaux représentants dans le domaine francophone de cette linguistique textuelle, est revenu sur la notion de «type de texte» au profit de celle de «séquence», unité de composition textuelle inférieure à l'ensemble représenté par le texte ${ }^{32}$, la transposition didactique lui a substitué celle de «forme de discours ${ }^{33}$ », au prix d'un risque de confusion avec d'autres acceptions comme celle de «types de discours» (discours littéraire, politique, scientifique, religieux...) ou encore celle de «formation discursive» qu'on doit à Foucault et qu'on retrouve dans le champ de l'Analyse du discours ${ }^{34}$.

30. «Pour le cycle central, l'accent est mis sur l'articulation et l'entrecroisement des formes de discours : en introduisant en $5^{\mathrm{e}}$ l'analyse du descriptif, on précise les liens entre le narratif et le descriptif, dont les fonctions réciproques pourront être approfondies en classe de $4^{\mathrm{e}}$ (avec la dimension explicative de la description, par exemple), et même en classe de $3^{\mathrm{e}}$ (avec sa dimension argumentative)», Coll., p. 86.

31. Voir ainsi la progression prévue, de la $6^{\mathrm{e}}$ à la $3^{\mathrm{e}}$, pour «Savoir argumenter», Coll., p. 87.

32. Voir son développement «En finir avec les “types de textes” » dans Linguistique textuelle. Des genres de discours aux textes, Nathan Université, I999, p. 8 I-84.

33. L'accompagnement des programmes précise que la classe de $3^{\mathrm{e}}$ ne doit pas être une «classe d'argumentation»: «Toutes les formes de discours y ont pleinement leur place, et c'est leur articulation que les élèves doivent apprendre à maîtriser. Les textes, du reste, ne correspondent que très rarement à la mise en œuvre de l'une d'entre elles; bien au contraire, elles s'y entrecroisent. C'est la raison pour laquelle on a évité de parler, dans les programmes, de «textes» argumentatif, descriptif, explicatif, etc., ouvrant à des typologies rigides, et qu'on a préféré la notion de discours, plus proche de la réalité vivante de la langue en acte. Il s'agit donc de comprendre les articulations des différentes formes de discours et d'apprécier, de manière souple, la façon dont elles s'agencent dans la réalité concrète des textes», Coll., p. 168 .

34. Pour une mise au point synthétique sur ces notions, voir R. Amossy, L'argumentation dans le discours. Discours politique, littérature d'idées, fiction, Nathan Université, 2000, p. I96-197. A. Boissinot, constatant cette tendance des documents d'accompagnement du collège de «tenter de neutraliser l'opposition et de renoncer à la distinction [entre texte et discours] en mettant en avant la notion de forme de discours», propose de maintenir une distinction mais à partir des notions de type et de genre, qui sont «deux angles d'attaque complémentaires sur la diversité des discours attestés». Pour éviter le «technicisme» (dérive qui fut, de fait, celle de la «lecture méthodique», introduite dans les programmes en i 987), A. Boissinot rappelle que travailler sur l'argumentation, «c'est aussi examiner comment certains procédés de type argumentatif sont pris dans des ensembles de discours plus vastes, plus complexes et qui 
Si on perçoit bien, dans les programmes de collège, comment l'approche par les formes de discours doit surtout permettre «l'introduction d'une grande variété de textes : textes porteurs de références culturelles, littérature, littérature de jeunesse, textes fonctionnels et documentaires, presse, bande dessinée, médias non verbaux» (Coll. p. 83), les genres étant définis comme des "pratiques discursives culturellement codifiées» qui «permettent d'appréhender des ensembles de textes d'un seul tenant», cette approche n'est plus un élément structurant des programmes du lycée et ne paraît subsister que pour mémoire dans la liste de notions déjà évoquée. Le terme de discours apparaît à propos de la perspective d'étude argumentative ainsi désignée : «L'argumentation et les effets de chaque discours sur ses destinataires.» Mais les accompagnements, dans leur introduction aux «Perspectives d'étude», reviennent en fait à la notion de texte : «Tout texte tient à une situation, relève d'un genre, tend à produire un effet sur ses lecteurs et auditeurs, fait écho à d'autres textes, s'en marque ou démarque, et bien sûr, met en jeu des registres d'expression et d'émotion» (Acc. p. I I-I 2). À propos de la mise en œuvre de l'objet d'étude «Démontrer, convaincre et persuader» en seconde, on trouve cette curieuse mise en relation de la notion de texte et de celle de discours :

La mise en relation du littéraire et du non-littéraire est un des éléments importants dans l'étude de l'argumentation. Elle permet en effet de manifester comment l'analyse de textes littéraires donne accès aux traits essentiels des discours et peut donc, a fortiori, être utile pour le non-littéraire. (Acc., p. 45)

Le terme de discours permet ici de caractériser le «non-littéraire» par opposition aux «textes littéraires». En dehors de ces quelques occurrences, il n'est guère question de discours dans les programmes et accompagnements du lycée, sinon à propos de l'étude de la langue pour distinguer trois niveaux : la phrase, le texte, le discours. Mais les développements consacrés au discours concernent les « régularités liées à l'énonciation» et ne disent rien des «formes de discours».

Les programmes du lycée font bien davantage référence à la notion de genre, les genres et registres étant une des quatre perspectives d'étude des textes. L'Accompagnement précise en outre, dans le développement qu'il consacre aux genres : «L'étude des genres constitue une part importante de l'enseignement du français au collège» (Acc., p. I4). C'est donc à l'étude des genres qu'il revient de distinguer des formes littéraires argumentatives. Certes, le texte officiel souligne les difficultés théoriques de la notion de genre, tout en

associent, selon des modalités diverses, le narratif, l'argumentatif, le descriptif, etc.» et il ajoute : «Il ne doit pas y avoir - ce serait un risque de sclérose - d'isolement et de réification du type de texte argumentatif conçu comme un objet d'étude en soi et isolé du reste» (art. cité, p. 6-7). 
précisant qu'il ne s'agit pas d'entrer dans de tels débats avec les lycéens mais «de leur faire acquérir une connaissance analytique des principaux genres de la littérature occidentale», c'est-à-dire des «quatre domaines généralement retenus : le récit, le théâtre, la poésie et la littérature d'idées» (Acc., p. I4). Les auteurs de l'Accompagnement conviennent que «ces notions risquent d'être trop générales et abstraites» et que la littérature d’idées, notamment, «est une catégorie vague et multiforme». Elle ne sera guère précisée néanmoins, sauf pour la classe de première où «au titre de l'étude de l'argumentation, sont abordés les genres de l'essai et du dialogue, les formes de l'apologue» (loc. cit.) $)^{35}$. Lorsqu'on sait la difficulté qu'il y a à définir l'essai, difficultés qui conduisent à se demander d'ailleurs si l'essai est bien un genre ${ }^{36}$, on ne peut s'empêcher de penser que cette précision n'en est pas vraiment une. Et quand on songe que l'apologue participe tout autant du récit, voire de la poésie, que de la littérature d'idées, on n'est guère plus avancé sur ce qu'il faut entendre par «littérature d'idées».

Cette catégorie «vague et multiforme» n'est nulle part vraiment interrogée ni analysée dans les accompagnements de programme où elle est présentée comme une sorte d'évidence qu'on déduit plutôt par soustraction du reste (ce qui n'est ni récit, ni théâtre, ni poésie). Elle réapparait à propos de l'objet d'étude consacré à l'argumentation en seconde sous le titre "Introduction à la littérature d'idées» et le sous-titre «Des débats marquants dans l'histoire culturelle» :

La réflexion sur les moyens et les finalités de l'argumentation se fondera sur des textes littéraires, sans pour autant exclure d'autres genres de textes et de documents. Elle introduit ainsi les élèves à la connaissance d'auteurs dont l'œuvre relève pour l'essentiel de la littérature dite «d'idées». Celle-ci est en elle-même un élément neuf pour les élèves au sortir du collège, où ils n'en ont eu que des aperçus. Cette approche de la littérature d'idées les initie progressivement, dès la seconde, à des réflexions qui feront ultérieurement l'objet de la philosophie. Cet ancrage dans la littérature, permettant de spécifier ce domaine en français par rapport aux sujets traités en éducation civique et sociale, justifie la forme des intitulés proposés ci-dessous : «Littérature et...». (Acc., p. 4I-42)

Les sujets de problématique suggérés sont les suivants : littérature et altérité, littérature et éducation, littérature et politique, littérature et guerre. Seul le dernier sujet est accompagné d'une liste qui renvoie partiellement à des genres : textes romanesques, pamphlets, lettres, articles de presse, documents visuels et cinématographiques. Le syntagme «littérature d'idées» est ainsi davantage

35. La partie de l'Accompagnement sur «L'argumentation en classe de première» y ajoute également la lettre ouverte «qui peut constituer alors une des formes que prend l'essai»! (p. 44). 36. Voir notamment P. Glaudes, J.-Fr. Louette, L’Essai, Hachette Supérieur, I 999. 
employé en seconde pour renvoyer à des thèmes (altérité, éducation, politique, guerre) qu'à des formes génériques spécifiquement argumentatives. On ne sait pas très bien non plus comment il faut entendre le terme «littérature» dans ce même syntagme : désigne-t-il le caractère «littéraire» des œuvres auxquelles il renvoie (littérarité d'ailleurs non définie), ou simplement un ensemble indéterminé de productions textuelles exprimant des idées, comme le suggère l'inclusion dans le corpus «d'autres genres de textes et de documents»? Les suggestions de textes faites pour les deux thèmes «Littérature et éducation» et «Littérature et altérité» ne nous éclairent guère : elles distinguent d'une part des «Textes littéraires» et d'autre part des «Documents». Mais pourquoi tel texte d'Érasme intitulé «Une philosophie de l'éducation» est-il rangé dans la catégorie des textes littéraires, tandis que celui de Fénelon, De l'éducation des filles, est considéré comme un document? Pourquoi encore les Réflexions sur la question juive de Sartre sont-elles un texte littéraire, et non Tristes tropiques de Lévi-Strauss, pour ne prendre que quelques exemples dans un classement qui laisse perplexe? On voit bien qu'il ne s'agit pas de réfléchir sur ce que pourrait être une «littérature d'idées», au sens littéraire du terme, mais bien plutôt de réunir des textes divers sous un thème donné, sans préoccupation de genre. Pourquoi pas d'ailleurs, si l'objectif est, comme il est dit, de préparer à la philosophie? Mais encore ne faudrait-il pas instrumentaliser ces textes, et donc les idées qu'ils développent, en les mettant uniquement au service de l'apprentissage de l'argumentation ${ }^{37}$.

Ce n'est que dans les développements consacrés à la classe de première qu'on voit s'ébaucher une réflexion sur le caractère littéraire de la «littérature que l'on appelle usuellement littérature d'idées» :

La mise en relation du littéraire et du non-littéraire est un des éléments importants dans l'étude de l'argumentation. Elle permet en effet de manifester comment l'analyse de textes littéraires donne accès aux traits essentiels des discours et peut donc, a fortiori, être utile pour le non-littéraire. Elle montre que la littérature d'idées permet de rendre compte des caractéristiques de tout texte argumentatif, mais aussi en quoi elle élabore des propos qui accèdent à la conviction, à la persuasion et au jugement en faisant intervenir l'art de plaire, toucher, émouvoir par la qualité même du texte. (Acc., p. 45)

37. J.-Fr. Massol a montré, dans son article «La littérature d'idées au temps de l'argumentation: quelle lecture pour l'apprenti philosophe et le futur citoyen?», que «l'approche de cette littérature à travers la perspective dominante de l'argumentation reste trop limitée» et «que l'on ne peut apprendre à comprendre des textes d'idées dans la seule saisie distanciée de leurs fonctionnements linguistiques et rhétoriques»: «Une telle position tend à produire, en effet, un pur délaissement des idées elles-mêmes, ce qui équivaut à un oubli du rôle que doit prendre la littérature qui les met en œuvre dans la formation des jeunes citoyens et dans l'initiation à la philosophie des élèves de seconde et de première.» Modernités. Le lecteur engagé. Critique-enseignement-politique, $\mathrm{n}^{\circ}$ 26, 2007 , p. 146. 
Entre approche de certains genres dits argumentatifs pour la classe de première et réservoir de problématiques en seconde et en première, la «littérature d'idées » a donc un statut bâtard et on peut penser qu'elle est surtout une étiquette commode qui permet de continuer à faire lire des grands auteurs du panthéon national comme Montaigne, Pascal, ou Diderot, qui trouvent mal leur place dans les autres objets d'étude du programme.

On retrouve avec la littérature d'idées l'absence de rigueur théorique qui caractérise la manière dont est introduite et présentée l'étude de l'argumentation dans les programmes et accompagnements du lycée. Sans doute est-ce ce flou théorique qui rend difficiles à cerner les apprentissages et leurs finalités tels qu'ils sont proposés dans les textes officiels.

\section{B. L'argumentation partout ... et nulle part}

\section{Diversité des objectifs de l'apprentissage de l'argumentation}

Les objectifs assignés à l'étude de l'argumentation sont en effet presque aussi variés que les approches : prendre en compte le point de vue d'autrui, faire une dissertation, adapter la communication au public, analyser un texte dit «argumentatif », raisonner, former le jugement critique, lire un essai, analyser les différentes formes de l'argumentation, débattre ou encore analyser une image publicitaire... Autant de savoir faire et de compétences qui relèveraient de l'étude de l'argumentation. Plus encore, l'argumentation se décline en savoir être ("prendre en compte la parole d'autrui», «accepter et comprendre la parole d'autrui», dans les programmes du collège, «apprendre à écouter l'autre», repris dans les accompagnements de programme du lycée $\left.{ }^{38}\right):$ la visée de son apprentissage est non seulement morale (amener au «respect d'autrui»), mais aussi sociale et politique puisque «la prise en compte d'autrui», comme le précisent les objectifs de la classe de troisième, est «envisagée à la fois dans sa dimension individuelle (dialogue, débat) et dans sa dimension sociale et culturelle» (Coll. p. I 53 ) et que le français participe «à la tâche générale d'éducation du citoyen» (Coll. p. I6). L'accompagnement du programme de troisième précise encore les valeurs en jeu dans l'argumentation :

Un de ces enjeux est de permettre aux élèves de mieux prendre place dans

l'espace de la discussion que le contexte contemporain, souvent décrit comme celui de la «crise des valeurs», ouvre largement. (Coll., p. 17I)

38. «Il s'agit d'apprendre à écouter l'autre, à nourrir de ce qu'il dit sa propre pensée pour la faire progresser, à choisir ses arguments en fonction de l'autre, non pour l'emporter, "trouver la faille", mais pour entrer dans le débat avec tout le respect d'autrui que cela suppose», dans «Démarches et progressions», Acc., p. 78. 
Ainsi, le premier objectif du travail avec les élèves au lycée sera «de leur faire maittriser l'enjeu fondamental de l'argumentation qui, donnant à la parole une chance contre la violence, est la régulation des conflits» (Acc., p. 2I). Vaste ambition que de mettre ainsi l'apprentissage de l'argumentation au service de la paix sociale! Et les accompagnements de programme soulignent encore à propos de l'apprentissage de la délibération en première que son «lien avec la formation du jugement et le respect des opinions d'autrui est ici capital pour la formation tant personnelle que civique, conformément aux finalités de l'enseignement du français au lycée» (Acc., p. 22). Si on ajoute que «les élèves sont sollicités par l'argumentation dans différentes disciplines : français, histoire, mathématique, sciences physiques, éducation civique, juridique et sociale, etc.» (Acc., p. 40) et que l'argumentation constitue de ce fait «un objet d'étude interdisciplinaire», on aura compris à quel point il est difficile de «circonscrire le domaine de l'argumentation» (loc. cit.).

De ce fait, l'argumentation dans les programmes se traduit dans beaucoup d'activités très différentes, écrites, orales, de lecture, d'écriture ou de prise de parole, dont l'énumération donne le tournis :

[...] le libellé des sujets doit préciser les situations d'énonciation et en diversifier les pratiques : justification d'une opinion sur un texte, interprétations, commentaires; lettres, éloges, critiques, articulation de points de vue différents sur un texte, un livre, un film, un sujet de société, préparation d'un entretien ou d'un débat; entretiens, débats, discours argumentés, travaux de groupes, etc. (Acc., p. 23)

L'argumentation [...] est étroitement liée à nombre de travaux écrits et oraux. Les exercices oraux de confrontation d'idées et de points de vue préparent au débat; le lien avec l'ECJS est manifeste. Les travaux écrits d'argumentation préparent à la dissertation. De même, des pages de textes littéraires argumentatifs peuvent faire l'objet d'un commentaire. L'écriture d'invention peut être aussi bien argumentative que figurative. (Acc., p. 42)

Diversité des pratiques d'écriture, diversité des exercices, mais aussi diversité des textes lus et étudiés puisque la perspective d'étude argumentative ou pragmatique permet d'aborder tout texte sous l'angle de son effet sur le destinataire.

\section{Un apprentissage de l'argumentation à travers des formes littéraires complexes.}

Dans une perspective pragmatique en effet, tout texte peut être considéré comme argumentatif puisqu'il «tend à produire un effet sur ses lecteurs et ses auditeurs». Et de fait, l'Accompagnement des programmes souligne à plusieurs reprises que «les faits argumentatifs sont omniprésents dans toutes sortes de discours» (p. 44), que l'argumentation «étant omniprésente dans les discours et les textes, elle doit aussi être présente tout au long de l'année» (p. 42). On la retrouve donc dans chaque objet d'étude ainsi que le précisent les développements suivants : 
Par ailleurs, l'argumentation peut être présente dans le théâtre, où elle est souvent un élément moteur des dialogues; elle l'est souvent aussi dans le roman (où les interventions du narrateur ont aussi un rôle important à cet égard). Enfin elle l'est de toute évidence dans l'éloge et le blâme. Elle doit donc être associée à ces différents objets d'étude. (Acc., p. 42)

En série littéraire, les deux objets d'étude spécifiques («Les réécritures», p. 54 et «L'épistolaire», p. 58) offrent de multiples cas d'approfondissement des analyses d'argumentation. En particulier, dans les réécritures, la citation constitue un argument (soit comme preuve, soit comme autorité) et la parodie est un moyen d'argumenter (par le rire critique, la connivence). Dans l'épistolaire, les liens entre argumentation et jeux de registres (le lyrisme comme moyen de toucher pour persuader; la lettre comme substitut du dialogue polémique) sont à mettre en avant. (Acc., p. 45)

À force d'être partout, l'argumentation risque bien de se retrouver nulle part. C'est pourquoi d'ailleurs devant ce risque de dilution, les textes officiels ont soin de préciser «qu'il est bon que soit consacré un temps spécifique [à l'argumentation] au cours de l'année» (Acc., p. 42) et que «l'omniprésence de l'argumentatif exige de répartir l'analyse de ses divers aspects» (Acc., p. 43) $)^{39}$. Et c'est la fonction des objets d'étude consacrés à l'argumentation de délimiter ce temps spécifique.

Néanmoins, les objets d'étude consacrés à l'argumentation ne permettent pas pour autant de préciser des genres spécifiquement argumentatifs, comme nous l'avons déjà vu à propos de la «littérature d'idées», en dehors de l'essai (dont on a rappelé les difficultés de définition) et du «dialogue d’idées» (appellation dont on pourrait aussi contester le caractère générique). On constate d'ailleurs que dans les manuels, toutes sortes de textes, relevant de genres très différents, sont enrégimentés pour l'étude de l'argumentation. L'extension du corpus argumentatif à la fiction et à la poésie est d'ailleurs rendue possible et encouragée par les instructions concernant ce que les programmes appellent «l'argumentation indirecte» qui doit être plus particulièrement étudiée en première. Cette notion d'«argumentation indirecte» semble être élaborée spécifiquement pour les programmes afin de ménager une place à l'apologue et de manière générale, à tout texte empruntant les voies de la fiction pour convaincre :

Plus largement, apologue qualifie des fictions ayant une visée argumentative, des fables, des contes, des exempla, aboutissant à une leçon morale (Acc., p. 44) ${ }^{40}$.

39. Et encore p. 44 : «Cependant, pour permettre aux élèves de saisir les différentes formes et enjeux de l'argumentation, il est recommandé qu'elle soit aussi, en cours d'année, l'élément central d'une séquence.»

40. À noter cependant qu'à deux pages de distance l'apologue est considéré d'abord comme relevant de l'argumentation directe comme l'essai et le dialogue ("Connaissances : étude de la littérature d'idées» p. 44), puis comme une «composante importante» de 
Elle est également mise en relation avec l'écriture d'invention. En effet, après avoir précisé les «deux dimensions majeures » que doit prendre l'étude de l'argumentation en première, à savoir «la délibération» et «l'argumentation indirecte, où une prise de position se manifeste moins dans un débat ouvert que par des voies visant à susciter une adhésion par l'agrément», le texte d'accompagnement précise que «l'exercice d'écriture d'invention est davantage lié» à cette deuxième dimension. L'examen des sujets de l'EAF conduite plus haut nous a montré que cette préconisation des programmes ne se retrouvait pas dans des sujets qui relèvent dans leur majorité de l'argumentation directe.

Comment comprendre cette tendance des programmes à retrouver l'argumentation dans presque tous les genres de texte, et leur insistance sur les formes d'argumentation indirecte en première? Paradoxalement, on veut apprendre aux élèves ce qu'est l'argumentation à travers ses formes les moins marquées et les plus complexes, en ayant fait l'économie des genres les plus clairement argumentatifs comme les discours politiques ou les plaidoyers et toutes les formes d'intervention directe dans le débat public. À l'œuvre depuis la fin du XIX ${ }^{e}$ siècle, la réduction du champ littéraire à des œuvres dont la finalité est perçue d'abord et avant tout comme esthétique a rendu difficile l'introduction dans les corpus scolaires d'œuvres dont la finalité est d'agir dans l'espace social et politique. La fable et le conte ont l'avantage de n'avoir jamais quitté le champ littéraire grâce à leur part de fiction. On pourra objecter que l'essai et le dialogue font partie des genres préconisés et que les programmes de seconde permettent d'aborder tous les genres argumentatifs. Mais l'essai est souvent d'un abord difficile, étant donné son ampleur et le caractère conceptuel de l'argumentation qui peut y être conduite (ce qui est aussi souvent le cas du dialogue philosophique). En outre les manuels, chargés de mettre en œuvre les programmes, se sont très peu emparés des possibilités ouvertes par l'étude de l'argumentation et ont eu tendance à recycler les mêmes extraits que par le passé en les abordant sous l'angle de l'argumentation.

\section{Imprécision des notions}

Alors qu'autant de responsabilités reposent sur l'apprentissage de l'argumentation, on serait en droit d'attendre des outils didactiques à la hauteur de tels enjeux. Or on a affaire, avec l'argumentation, à un champ scientifique encore très récent et à des recherches d'origines très diverses. Les programmes ont tenté la transposition didactique de notions et d'un vocabulaire qui

l'argumentation «dans ses formes indirectes» («Mise en œuvre», p. 45). Autre cas d'«argumentation indirecte» signalé à côté des apologues : la "part de l'apologie dans le biographique», Acc., p. 23. 
sont encore loin d'être stabilisés, au contraire de ce qu'on observe, par exemple, en narratologie. Le résultat est donc décevant.

Alors que les programmes reprennent à Perelman sa définition de l'argumentation comme «forme de discours qui vise à obtenir l'adhésion d'un auditeur ou d'un lecteur aux thèses qu'on présente à son assentiment» (Acc., p. 2I), ils ne transposent pas sa classification des arguments, c'est-à-dire ce qu'il appelle en fait des «schèmes argumentatifs» et qui sont une nouvelle tentative de formalisation des lieux ou topoï de la rhétorique antique (TA, p. 252). De ce fait, la définition des arguments donnée dans les accompagnements se contente d'une formulation qui relève davantage du sens commun que de la clarification théorique : «Les arguments sont alors des raisons plus ou moins fortes autour d'une thèse proposée» (Acc., p. 4I). Tous les mots utilisés dans la définition (thèse, raisons, et donc arguments) restent problématiques dès qu'on veut les appliquer, par exemple, à l'analyse des textes et sont donc peu opérationnels sur le plan didactique.

Pour comprendre cette difficulté tout à la fois terminologique et notionnelle, il faut remonter aux théories de l'argumentation existantes. J'ai déjà évoqué Perelman, mais il est une autre référence majeure pour les théories de l'argumentation : c'est celle du philosophe Stephen E. Toulmin qui publie en I958, la même année que le Traité de l'argumentation, The Uses of Argument ${ }^{41}$. Je ne retiendrai ici de Toulmin que son modèle de l'argument, partie très restreinte de sa théorie, non pour la simplifier et la réduire, mais parce que le modèle a pu inspirer des applications à l'analyse des textes et des tentatives de transposition didactique ${ }^{42}$. Ainsi Jean-Michel Adam s'est inspiré de ce modèle pour l'établissement de sa séquence argumentative prototypique ${ }^{43}$. Reste que des modèles comme celui de Jean-Michel Adam, formellement très lourds, ne sont guère opérationnels au-delà de l'étude de micro-séquences et qu'à l'échelle d'un texte entier, ils deviennent très difficiles à manier ${ }^{44}$. Si on y ajoute les variations terminologiques dues aux différentes traductions (warrant traduit tantôt par «loi de passage», tantôt par «garanties», backing par

4I. Cambridge University Press, trad. française Les Usages de l'argumentation, PUF, I 993.

42. Pour une présentation plus complète, voir par exemple Ph. Breton, G. Gauthier, Histoire des théories de l'argumentation, La Découverte et Syros «Repères», 2000, p. 54-68.

43. Voir La Linguistique textuelle. Introduction à l'analyse textuelle des discours, Armand Colin «Cursus», 2005, p. I 57-I62.

44. L'espace de cet article ne me permettant pas de développer plus avant l'analyse de ces modèles, je renvoie à l'article de C. Masseron, «Pour une didactique de l'argumentation (écrite) : problèmes, objets et propositions (I)» paru dans Pratiques, $\mathrm{n}^{\circ}$ 96, décembre 1997 , p. 7-34. Elle analyse quelques schémas décrivant le discours argumentatif et en tire des conclusions pour la didactique du français. 
«support», «fondement» ou encore «étayage»), on mesure la difficulté de transposition didactique d'un tel modèle.

De manière générale, les outils d'analyse ne sont pas stabilisés dans le champ didactique et conduisent, dans les programmes, à un bricolage notionnel. On en prendra pour exemple cet extrait des accompagnements des programmes de troisième consacré aux moyens linguistiques de l'argumentation :

- Schéma argumentatif. Les élèves ont depuis longtemps appris à reconnaittre et à manipuler le schéma narratif. On les initie en $3^{\mathrm{e}}$ à la reconnaissance et à la manipulation d'un schéma argumentatif à travers quelques circuits argumentatifs simples : la transformation très générale qui conduit d'une thèse refusée à une thèse proposée, la combinaison des arguments et des exemples, la structure canonique de base (thèse, antithèse, synthèse) et son dépassement dans la réalité des textes, l'emploi de quelques connecteurs argumentatifs susceptibles de segmenter un déroulement argumentatif («d'une part..., d'autre part», «mais», «par ailleurs», «en outre», «en revanche», «parce que», «puisque»). (Coll., p. 170)

On voit bien ici comment il s'agit de fabriquer la notion de «schéma argumentatif » par analogie avec celle de «schéma narratif», alors même qu'il n'existe pas de définition claire et univoque de ce que pourrait être «le schéma argumentatif» (si tant est qu'il en existe un). Quant à l'expression «circuit argumentatif», qui paraît désigner ici une unité inférieure à celle du «schéma argumentatif ", elle renvoie à des configurations extrêmement diverses et dont la définition est renvoyée à l'usage commun (mais qu'est-ce précisément qu'une «thèse $»^{45}$ ?). Sans doute faut-il rechercher l'origine de cette expression de «circuit argumentatif » dans l'ouvrage déjà cité d'Alain Boissinot, Les Textes argumentatifs, où le "circuit argumentatif », qui désigne l'agencement des arguments, fait partie d'un ensemble de caractéristiques générales censées définir les textes argumentatifs (par exemple la polyphonie énonciative ou la présence de connecteurs) mais qui, en réalité, sont communes à bien des textes $^{46}$. Si les programmes de lycée n'évoquent plus la notion problématique de «texte argumentatif» en tant que type de texte, on retrouve, je l'ai déjà

45. On pourrait aussi, si le constat n'était pas déjà aussi accablant, montrer à quel point la liste des termes désignés par le syntagme «connecteurs argumentatifs» est hétérogène et renvoie à des faits linguistiques qui ne sont pas du même ordre.

46. Cette tentative pour définir un type de texte argumentatif renvoie bien sûr aux typologies textuelles héritées de la linguistique, déjà évoquées plus haut, mais également à la lecture méthodique, modalité d'analyse des textes destinée à remplacer l'explication de texte à partir des programmes de 1988 : fondée notamment sur un relevé objectif des marques linguistiques de surface, elle faisait explicitement référence à une typologie des textes qui justifiait des méthodes de lecture diversifiées. C'est cette approche qui est à l'origine de la nouvelle épreuve du baccalauréat introduite en 1995 (Bulletin officiel spécial nº Io, du 28 juillet I994) qui comportait comme premier sujet l'«étude d'un texte argumentatif». 
souligné, la recommandation de travailler à propos de l'étude raisonnée de la langue sur les «liens logiques ou apparemment logiques» en seconde et sur la modalisation en première (Acc., p. 74), héritage de ces tentatives typologiques.

Arrêtons-nous pour finir sur une autre notion problématique : celle de «délibération» qui tient une grande place dans les programmes du lycée puisqu'elle structure l'objet d'étude de première consacré à l'argumentation : «Convaincre, persuader, délibérer». La notion a vraisemblablement été empruntée à Perelman: dans le chapitre du Traité de l'argumentation consacré aux «Cadres de l'argumentation» (où l'on retrouve la distinction entre convaincre et persuader qu'il établit à partir de la distinction entre auditoire universel et auditoire particulier), il traite de l'auditoire universel, puis de l'argumentation devant un seul auditeur et enfin de la délibération avec soimême (TA, p. 53-59). Il considère en effet «qu'il y a tout intérêt à considérer la délibération intime comme une espèce particulière d'argumentation» et ajoute que «c'est l'analyse de l'argumentation adressée à autrui qui nous fera mieux comprendre la délibération avec soi-même» (p. 54). Étant donné ce que doivent les programmes aux catégories de Perelman, il n'y aurait rien d'étonnant à ce que la délibération intime y figure. Mais la notion de délibération fluctue curieusement dans les textes officiels et finit par devenir un concept protéiforme dont on se demande s'il n'a pas été élaboré pour les besoins de la cause. Ainsi, lorsqu'il est question, dans la partie de l'Accompagnement consacrée aux perspectives d'étude, de la progression de la seconde à la première en ce qui concerne "Argumentation et délibération», la délibération est présentée comme une des deux dimensions majeures de l'argumentation (avec l'argumentation indirecte) : elle est ce «qui correspond au cas le plus abouti où la confrontation d'idées et de prises de position débouche sur un jugement (individuel et collectif)» (Acc., p. 2I). Il ne semble pas que cette définition corresponde au cas de la délibération intime telle que la conçoit Perelman. Plus troublant encore : un peu plus loin le texte officiel tente un rapprochement avec les trois grands domaines de l'argumentation tels que les avait définis la rhétorique antique. Il traite ainsi du genre délibératif :

- Le délibératif, qui concerne la discussion sur l'avenir, sur ce qui n'est pas encore réalisé, sur ce que l'on projette d'organiser; ce qui renvoie aussi bien au débat politique (on pourra travailler ce point en ECJS) qu'à la discussion quotidienne, dès qu'on se trouve confronté, seul ou collectivement, à une décision. Le travail sur le théâtre (monologue intérieur, scène de confrontation d'opinions sur une décision à prendre) est un moyen d'aborder cette réflexion tout en préparant les élèves à la classe de première, où ils devront étudier l'essai. (Acc., p. 22) 
On glisse ainsi de la catégorie rhétorique du délibératif (elle-même définie de manière très large) à la «discussion quotidienne» puis au «monologue intérieur». Si l'on doutait que le délibératif et la délibération ne font qu'un ou plutôt que le délibératif n'est qu'un prétexte pour amener à la délibération, il suffit de lire le paragraphe suivant consacré à la classe de première :

Selon la progression indiquée par le programme, la délibération constitue un des enjeux majeurs de la classe de première. Ce terme désigne les situations où sont pris en compte plusieurs points de vue afin de construire un jugement. Il ne doit pas être entendu en son sens restreint et technique (délibération d'une assemblée, d'un jury) mais dans sa globalité (ainsi les «Stances» de Rodrigue dans $L e$ Cid sont un exemple frappant de délibération personnelle, la «tempête sous un crâne» de Jean Valjean dans Les Misérables de même...). (loc. cit.)

Ainsi, cet «enjeu majeur de la classe de première» est-il défini de façon très large, au point qu'on ne voit plus très bien ce que la délibération désigne, hormis lorsqu'elle prend le sens restreint qu'elle peut avoir au théâtre et que semble désigner l'exemple donné entre parenthèses des stances du Cid:

Délibération [...] Scène où le personnage évoque un conflit intérieur déchirant (d'origine politique souvent), en un monologue ou dans des stances, en s'efforçant de prendre une décision, parfois aidé par ses conseillers. L'orateur expose ses motivations et ses arguments, hésite longuement ou s'arrange pour choisir la moins mauvaise solution ${ }^{47}$.

On comprendra mieux quel est cet «enjeu majeur» lorsqu'on aura perçu ce qui se cache en fait derrière cette notion de délibération, à savoir la dissertation. En effet, il est clairement dit, à propos de la délibération en première, que l'exercice de la dissertation lui «est étroitement lié» tandis que l'écriture d'invention «est davantage liée» à l'argumentation (Acc., p. 22 et $43^{48}$. Dans la partie de l'Accompagnement consacrée à la dissertation, celle-ci est définie avant tout comme «un exercice de délibération - elle confronte des arguments divers, voire divergents». Elle suppose le «respect de l'opinion proposée» et donc le «respect de la voix de l'autre»; elle «est ainsi un apprentissage de l'esprit critique» et «enfin un apprentissage de la délibération réfléchie» qui «débouche sur une prise de position» (Acc., p. 96). Et sous le titre «Dissertation, argumentation et délibération», le texte officiel insiste encore :

La démarche fondamentale de la dissertation est bien une démarche de délibération : construire un jugement en prenant en compte plusieurs points de vue et

47. Voir P. Pavis, Dictionnaire du théâtre, Messidor-Éditions sociales, I 987 , p. I I 3.

48. Et dans les Programmes: «La classe de seconde met surtout en lumière les façons de convaincre et persuader; en classe de première, on insiste sur les formes et pratiques liées à la délibération; entre autres exercices, la formation à la dissertation concourt à cette fin.», «Préambule : le français au lycée», II, p. I I. 
définir ce jugement non comme un absolu, mais comme un choix que l'on fait en connaissance de cause parmi plusieurs façons de voir, correspond bien à la réflexion délibérative.

Si l'on comprend que tout converge ainsi vers l'exercice suprême que représente la dissertation, enjeu majeur de la classe de première et donc de toute la scolarité, comme celui du discours l'avait été dans le passé lorsque la rhétorique régnait sur l'enseignement, alors on peut s'expliquer comment a été fabriquée la notion de délibération, à partir de la dissertation, et non à partir de pratiques sociales de référence ou en s'inspirant de la tradition rhétorique. Certes, on peut comprendre ce souci légitime d'établir des liens entre les différentes parties des programmes et cette volonté d'établir une cohérence autour de certaines notions. Mais à vouloir réunir sous une étiquette unique le débat politique, le monologue intérieur et la dissertation, on court le risque de tout brouiller et on ne voit pas, en fin de compte, ce qu'apporte aux programmes le mot de «délibération». Le risque est plutôt de diluer le sens de notions bien définies par la tradition rhétorique, au lieu de les mettre au service de la didactique de l'argumentation.

\section{Un retour non assumé à la rhétorique}

Comme on vient de le voir à propos de la notion de délibération, les textes officiels ne se fondent pas seulement sur la «nouvelle rhétorique» de Perelman. Aux fondements théoriques déjà examinés plus haut, il faut en effet ajouter celui de la rhétorique, explicitement évoquée dans un développement des accompagnements intitulé «Contenus et objets d'étude : argumentation, littérature d'idées et rhétorique». Sont rapidement survolées un certain nombre de notions rhétoriques : enthymème (introduit comme par effraction), topoï (assimilés aux clichés et aux stéréotypes), ethos et pathos, notions fondamentales qui ne sont pas mises en relation avec les développements consacrés à «convaincre» et «persuader» alors qu'on aurait pu attendre qu'elles le soient ${ }^{4}$. Il est demandé également «de faire découvrir aux élèves les étapes (le mot «parties» est impropre en ce cas) de l'élaboration de tout discours, notamment celle de l'inventio/invention» (Acc., p. 23). Il pourrait être intéressant effectivement de travailler avec les élèves sur ce que la rhétorique

49. En rhétorique en effet, l'ethos et le pathos font partie de l'inventio et donc de la recherche des moyens de convaincre auxquels il faut ajouter les faits et les raisonnements (que certains regroupent parfois sous le nom de logos). Ces trois ensembles de moyens se trouvent chez Cicéron avec les trois buts qu'il assigne à l'orateur : docere (qui correspondait au logos), conciliare ou delectare (l'ethos), movere (le pathos). Bien mieux que la distinction convaincre-persuader, ces trois verbes permettent de comprendre par quels moyens l'orateur ou l'auteur entend agir sur un auditoire ou son public. 
désignait par inventio et notamment sur les différents types de preuves, en lien avec l'ethos et le pathos. Mais nous avons vu qu'il était seulement question dans l'Accompagnement, de manière très sommaire, des "grandes formes de raisonnement» qui ne relèvent que très partiellement de la rhétorique, et ce n'est qu'à propos de l'écriture d'invention que le texte officiel revient sur «la notion d'invention» (p. 90) et rappelle le sens rhétorique de dispositio, inventio et elocutio. Mais alors qu'il a été demandé plus haut (p. 23) d'initier les élèves au travail de l'inventio/invention au sens rhétorique du terme, la suite du développement brouille les cartes en redéfinissant le terme «invention» dans l'expression «écriture d'invention»:

L'écriture d'invention est donc, comme il a été vu, invention au sens entier du terme : inventio mais aussi choix de dispositio en fonction d'une situation, et elocutio; mais elle est encore - au sens où invention est entendu comme synonyme de découverte - une entreprise d'exploration du langage, de ses ressources et de ses contraintes. (Acc., p. 90)

Le texte officiel, après avoir prétendu revenir à une notion rhétorique, la redéfinit comme bon lui semble en prétendant inscrire le terme dans une filiation rhétorique alors qu'on a manifestement affaire à deux emplois différents du mot «invention».

Le plus surprenant concerne cependant le traitement réservé aux genres traditionnels de la rhétorique : délibératif, judiciaire, épidictique. Leur définition est tout d'abord tronquée : des catégories d'Aristote, les accompagnements de programme ne gardent que celle du temps (le délibératif concerne l'avenir, le judiciaire le passé) et ignorent les lieux et institutions (assemblée délibérative, tribunal, assemblée de citoyens), et donc les auditoires, concernés par ces genres. Pour aucun d'entre eux il n'est rappelé qu'ils désignaient des discours prononcés en public. Ainsi, le genre épidictique, discours de commémoration ou d'apparat réunissant une communauté de citoyens, est défini par «la fonction d'émouvoir le récepteur à partir d'une réalité connue» (Acc., p. 22). La préoccupation essentielle des accompagnements de programme parait être de rabattre les genres rhétorique sur des genres littéraires qui peuvent sembler leur correspondre. Le délibératif est ainsi renvoyé au théâtre, comme nous l'avons déjà vu, mais sans évoquer sa dimension politique. Le plus étonnant concerne le genre judiciaire qui fait l'objet de rapprochements particulièrement incongrus :

- Le judiciaire, qui vise la connaissance de la vérité effective de ce qui s'est passé. Au-delà du seul univers juridique, ce genre prévaut dans le journalisme d'investigation et dans tous les récits qui prennent la forme d'une enquête (voir le roman policier). On pourra travailler cet aspect en relation avec les séances consacrées au récit. (Acc., p. 22) 
Un vague rapprochement thématique (justice, donc procès, donc enquête) permet de glisser vers des genres dont on se demande ce qu'ils désignent (quid du «journalisme d'investigation»?) ou qui n'ont plus qu'un rapport très partiel et biaisé avec l'argumentation (le roman policier). Comme si les genres du réquisitoire et du plaidoyer n'existaient plus de nos jours et comme si on ne pouvait pas en trouver d'exemples dans la littérature ${ }^{5}$ !

Quant à l'épidictique, on comprend mieux pourquoi, alors même que c'est le genre dont la visée argumentative est la plus problématique pour les élèves, il est le seul rescapé des trois genres traditionnels sous la forme de l'objet d'étude «L'éloge et le blâme» puisqu'il est dit que «La tradition littéraire offre de nombreux exemples de ce genre : oraison funèbre, apologie, dithyrambe, panégyrique, blason, portrait, mais aussi pamphlet, diatribe, satire...». Il est surtout un cadre très commode pour y glisser l'étude du portrait dans des œuvres qui n'ont plus rien à voir avec le genre épidictique de la rhétorique ${ }^{\text {I }}$. Une «Remarque» développée dans l'Accompagnement des programmes (p. $5 \mathrm{I}$ ) justifie le choix de l'épidictique parce que «historiquement, [il] constitue le carrefour de ces deux domaines de la production littéraire» que sont la poétique et la rhétorique. Cette justification simplificatrice et contestable (qui tient sans doute au fait que traditionnellement, la valeur qui gouverne l'épidictique est le beau) montre en fait que la rhétorique a bien du mal à revenir dans les programmes et qu'on ne consent à en accepter que ce qui peut s'acclimater dans la représentation dominante d'une littérature surtout vouée au beau et au plaisir désintéressé ou dont l'engagement passe par des formes reconnues comme littéraires comme la poésie, le théâtre ou le roman ${ }^{22}$. On voit mal

50. Très curieusement pourtant, on les retrouve dans la liste des «Genres et formes de l'éloge et du blâme», le plaidoyer du côté des genres de l'éloge, le réquisitoire de celui des genres du blâme! Éloge et blâme sont ainsi devenus des catégories fourre-tout qui n'ont plus qu'un rapport très lointain avec l'épidictique, comme le montre la liste hétérogène des genres qui sont censés en relever (voir p. 5 I des Accompagnements).

51. Voir la liste d'exemples donnée p. 52 des Accompagnements à la suite du développement sur l'objet d'étude. Il est dit également p. 5 I : «Le lien est fort avec l'écriture d'invention, notamment lors d'un travail sur le portrait.» Là encore, on trouvera peu d'exemples, dans les sujets de l'EAF, d'écriture d'invention sur le portrait, et encore moins dans une perspective épidictique.

52. C'est aussi la trace d'une certaine façon de réécrire l'histoire de la rhétorique et celle de la littérature qui serait née d'une opposition avec le langage utilitaire, qui trouve sa justification en dehors de lui-même, tandis que la littérature est à elle-même sa propre finalité. Dans cette perspective, l'épidictique, qui vise le beau, ne peut que préfigurer la parole inutile admirée pour elle-même. Sur le rôle de T. Todorov et de sa Théorie du symbole (1977) dans l'invention d'une histoire de la rhétorique qui relie directement le Dialogue des orateurs de Tacite au manuel de Fontanier, voir ma thèse, L'Éloquence parlementaire sous la monarchie de Juillet. Guizot, Thiers, Tocqueville, Grenoble 3, 2000. Voir également l'article d'A. Boissinot déjà cité p. $8-9$. 


\section{ÉCRITURE D'INVENTION ET ARGUMENTATION}

comment, dans ces conditions, l'étude de l'éloge et du blâme répondrait au «besoin de former la personne et le citoyen» dont l'urgence actuelle est proclamée par la même Remarque. En continuant en outre à véhiculer la thèse, déjà invalidée au moment de la parution des programmes ${ }^{33}$, d'une rhétorique qui se serait trouvée restreinte aux figures au XIX ${ }^{\mathrm{e}}$ siècle ${ }^{54}$, l'Accompagnement se montre en retard sur le renouveau des études rhétoriques et de surcroit, ne peut guère mettre en cohérence les choix des objets d'étude avec les orientations de la «nouvelle rhétorique» perelmanienne, ancrée dans le droit, la politique et la philosophie, «nouvelle rhétorique» qui a pourtant inspiré une partie des programmes.

Par ailleurs, cette influence de Perelman peut rendre difficile la prise en compte de l'héritage rhétorique. Nous avons vu en effet que les positions rationalistes de Perelman l'avaient poussé à exclure du champ de sa réflexion toute la partie de la rhétorique consacrée aux passions et nous avons vu également que la distinction convaincre-persuader, qui structure les objets d'étude du lycée, entretenait la dichotomie entre raison et affects :

Le but est de percevoir et comprendre les différences, mais aussi les liens, entre démontrer - dans le domaine des vérités vérifiables - et convaincre ou persuader, en s'appuyant sur des arguments rationnels ou sur des facteurs affectifs (Progr., p. $14^{\text {ss) }}$.

Ainsi formulée, et alors que notre tradition cartésienne reste marquée par une vieille méfiance à l'égard des passions en particulier et de la rhétorique en général, cette distinction ne peut permettre de penser la notion de pathos comme moyen de preuve ni la distinction cicéronienne des buts de l'orateur: docere, conciliare ou delectare, movere ${ }^{\varsigma}$. Évoquant une argumentation tendue «entre

53. Voir notamment Fr. Douay, «La rhétorique en France au XIX ${ }^{\mathrm{e}}$ siècle», Histoire de la rhétorique dans l'Europe moderne, M. Fumaroli éd., PUF, I999, et A. Vibert, «Fontanier : autour et au-delà. La rhétorique dans le premier tiers du XIX ${ }^{\mathrm{e}}$ siècle», RHLF, $2005, \mathrm{n}^{\circ} 2$.

54. La rhétorique «a fait l'objet, au XIX ${ }^{\mathrm{e}}$ siècle, d'une sclérose dans son enseignement, qui l'a réduite à peu près aux seules figures de style» et «une partie de la tradition littéraire s'est détachée de la tradition éloquente», Acc., p. 5 I.

55. Voir aussi : «Les démarches de la conviction relèvent de la raison, du dialogue et de l'échange; elles sollicitent le savoir. [...] La persuasion relève davantage que la conviction de la suggestion, de la séduction, de la tentation; elle sollicite moins le savoir que le désir ou la crainte», Acc., p. 4I.

56. Un passage de l'Accompagnement qui concerne la littérature d'idées tente de conjuguer ces différents buts : «[L'étude de l'argumentation] montre que la littérature d'idées permet de rendre compte des caractéristiques de tout texte argumentatif, mais aussi en quoi elle élabore des propos qui accèdent à la conviction, à la persuasion et au jugement en faisant intervenir l'art de plaire, toucher, émouvoir, par la qualité même du texte.» (p. 45). Mais on sent bien qu'ici «l'art de plaire, toucher, émouvoir» est conçu davantage comme une sorte de supplément de «qualité» de la littérature que comme une réflexion sur les différents moyens dont dispose un auteur pour convaincre. 
la sécurité rationnelle de la preuve et le risque de la manipulation trompeuse» (Acc., p. 4I), les textes officiels dessinent ainsi un système de valeur implicite où la «bonne» argumentation serait du côté du convaincre et la «mauvaise» du côté du persuader (en témoigne notamment l'exemple de la publicité donné pour illustrer la persuasion p. 4I). Mais ce système se complique encore par la «récupération» du persuader du côté de l' "argumentation indirecte» qui en fait alors un objet littéraire acceptable et même recommandable, au nom cette fois de critères de plaisir :

Persuader se fait souvent non seulement par une argumentation directe, affichée comme telle, mais aussi par une argumentation indirecte, sous le voile du récit, du dialogue, de l'agrément ou du divertissement [...]. (loc. cit.)

C'est ainsi qu'on retrouve une certaine conception de la rhétorique réduite cette fois à un art d'agrément et non conçue comme un moyen d'agir par la parole.

Dans leur volonté de renouer les liens avec la tradition rhétorique rejetée à la fin du XIX ${ }^{\mathrm{e}}$ siècle, les programmes sont donc restés à mi-chemin de leur entreprise de réhabilitation, partagés entre les théories de Perelman, la poétique des genres issue du rejet de la rhétorique et une méfiance toujours sensible pour cette discipline : tendance à la manipulation, relativisme idéologique, le vieux procès fait à la rhétorique est toujours à l'arrière-plan, mais c'est plus encore son lien avec la vie de la cité qui dérange une institution scolaire plus encline à débattre de questions esthétiques que de questions politiques.

Les compromis théoriques obligés, mais aussi les approximations et les difficultés d'harmonisation entre des notions très différentes dues à la transposition de «savoirs savants» hétérogènes sur l'argumentation expliquent donc en grande partie la tendance à retrouver dans les sujets d'invention du baccalauréat le modèle des genres scolaires éprouvés de la dissertation et du commentaire. Quelles pistes d'apprentissage pourrait-on proposer pour une écriture argumentative réellement différente de ce modèle? 


\section{Pour une didactique des genres argumentatifs non scolaires}

\section{A. Pour une délimitation claire des genres argumentatifs}

Il faut d'abord se délivrer du «tout argumentatif » qui conduit à envisager n'importe quel texte dans une perspective argumentative ${ }^{57}$. Il ne s'agit pas de discuter ici les approches théoriques de l'argumentation dans la langue ou, de manière plus générale, de réfléchir à la portée illocutoire de tout discours. Ainsi que l'écrit Jean-Michel Adam :

L'hypothèse du «tout argumentatif » est [...] juste au niveau de la théorie pragmatique générale : un récit, une explication ont très souvent pour fonction globale d'argumenter. Comme le dit Jean-Blaise Grize : «tout discours peut être une argumentation», mais il ajoute «mais en lui-même $[\ldots]$ un énoncé n'est ni argumentatif, ni non argumentatif». Nous sommes d'accord pour dire que «le caractère argumentatif d'un discours repose avant tout sur la finalité de celui qui le produit $^{88}{ }^{8}$.

Je ne suivrai pas pour autant Jean-Michel Adam dans sa décision de limiter son propos à la séquence et à la phrase périodique argumentatives. Les formalisations qu'il propose sont en effet très complexes et difficiles à utiliser pour des textes d'une certaine ampleur. Je me situerai dans une perspective didactique qui vise à élire des genres dont la finalité première est de convaincre leurs destinataires en modifiant leurs idées et représentations, avec généralement une volonté d'influer sur leurs actions.

Reste cependant à préciser ces genres. La liste de «travaux écrits et oraux» que proposent les accompagnements de programme pour l'objet d'étude argumentation est en effet très large et hétérogène, puisqu'elle mêle genres proprement dits, pratiques de classe et compétences argumentatives et qu'elle est placée sous le signe de la «diversification» dans le but, annoncé lors des présentations des programmes, d'amener les enseignants à former les élèves de seconde sans se référer systématiquement aux exercices du baccalauréat:

57. Cette approche argumentative ne se limite d'ailleurs pas aux textes. On trouvera par exemple dans le numéro 927 de janvier 2007 des Textes et documents pour la classe, intitulé «Argumenter», l'exemple d'une séquence consacrée à «Art et argumentation» dont la première séance est consacrée à mettre en évidence la "dimension argumentative» d'une vanité (S. Stoskopff, Grande Vanité, I64I) et à reformuler les intentions du peintre «en reprenant les termes du quatrain [qui accompagnent le tableau] et en y ajoutant des connecteurs qui fassent apparaître nettement la logique de sa démarche pour mettre en place de façon inductive l'articulation entre le point de vue et la stratégie argumentative»! (p. 43, c'est moi qui souligne). Un tel non-sens se passe de commentaire.

58. «Une approche textuelle de l'argumentation», dans L'argumentation aujourd'bui. Positions théoriques en confrontation, op. cit., p. 79. Les deux citations de J.-B. Grize sont tirées de Logique naturelle et communications, PUF, I996, p. I 8 et 19. 
[...] le libellé des sujets doit préciser les situations d'énonciation et en diversifier les pratiques : justification d'une opinion sur un texte, interprétations, commentaires; lettres, éloges, critiques, articulation de points de vue différents sur un texte, un livre, un film, un sujet de société, préparation d'un entretien ou d'un débat; entretiens, débats, discours argumentés, travaux de groupes, etc. (Acc., p. 23)

Pour la première, en revanche, les programmes se concentrent, nous l'avons vu, sur l'essai, le dialogue d'idées et l'apologue, et ajoutent encore la lettre ouverte (Acc., p. 44). Mais d'une part ces genres intéressent les auteurs des programmes davantage pour leur contribution à la formation du jugement qu'à l'art de persuader ${ }^{\Im 9}$. D'autre part, ils sont présentés surtout du point de vue de l'étude des textes et très peu de celui de l'écriture d'invention. À propos de l'écriture d'invention proprement dite, les accompagnements de programme sont prolixes sur les types d'exercice à mettre en œuvre en seconde concernant transformation et transposition, imitation et reprise et enfin les «germinations». Mais ils le sont beaucoup moins pour les exercices consacrés à l'écriture d'invention argumentative (voir Acc., p. 94). On remarquera que ces instructions n'abordent pas l'écriture d'invention argumentative d'un point de vue générique et que les exercices proposés ne se retrouvent pratiquement pas dans les sujets d'invention de l'EAF (à quelques exceptions près pour les exercices présentés sous le chapeau «Confirmer ou réfuter»).

Pour la première, on trouve une curieuse répartition des sujets entre dissertation et écriture d'invention selon la position que l'on souhaite prendre dans un débat d'idées : si l'on a un point de vue tranché, et qu'on peut «aboutir à un jugement qui vaut décision», on est «dans la logique de la dissertation»; si deux points de vue restent en balance, ou qu'on a une préférence peu marquée, on est plutôt dans celle du dialogue; si l'on ne peut "prendre en compte qu'un point de vue principal, la logique est alors davantage celle de la lettre ou de l'essai (éventuellement sous forme d'article)» (Acc., p. 94). Le choix même du sujet est alors conçu comme un «exercice de jugement» et la capacité à choisir la forme d'écriture à employer en fonction du contenu comme un but de la classe de première. On s'interrogera d'abord sur cette inversion paradoxale qui consiste à faire de la dissertation un genre qui débouche sur

59. «Des genres proprement argumentatifs sont étudiés : ils doivent permettre de discerner des cas où les textes montrent un jugement qui prend forme, ou qui a pris forme et s'affirme (certains essais, certains dialogues), des cas où il est en suspens (autres cas de dialogues, voire d'essais), des cas d'argumentation indirecte (apologues, part de l'apologie dans le biographique).», Acc., p. 23. Voir également ce qui est dit de l'essai : «Genre littéraire, l'essai se distingue d'autres textes argumentatifs puisqu'ici le langage ne sert pas qu'à transmettre une pensée, il la donne à saisir dans son élaboration même : le mot [...] se rattache par son étymologie à l'exercice de la pensée», Acc., p. 44. 
une «décision» et donc sur l'action, alors que le dialogue, la lettre, et l'essai sont renvoyés à une mise en œuvre du jugement qui semble rester purement spéculative et en tout cas éloignée de toute finalité pragmatique. Si on comprend bien ensuite la volonté de faire concevoir une forme d'écriture qui serait en rapport avec un contenu de pensée, il ne peut qu'y avoir un décalage avec les pratiques de la classe qui laissent rarement aux élèves la possibilité de réfléchir sur le choix d'une forme d'écriture et leur proposent le plus souvent une consigne qu'ils n'ont pas eue à élaborer. Enfin, si la simplification peut avoir des vertus didactiques, cette répartition des rôles entre dissertation, dialogue et lettre ou essai procède d'une réduction simpliste des caractéristiques de chaque genre et ne peut être d'un grand profit didactique ${ }^{60}$.

Si l'on se tourne maintenant vers les instructions concernant l'écriture d'invention à l'épreuve écrite de français du baccalauréat, la définition des genres argumentatifs parait plus précise, en particulier dans la note de service de 2006 qui a modifié les instructions initiales de $200 \mathrm{I}^{61}$. La note distingue l'écriture d'invention qui «peut s'exercer dans un cadre argumentatif » et celle qui «concerne le genre narratif», là où le texte précédent disait simplement qu'elle «se fonde sur les contraintes littéraires des genres inscrits au programme de première» et «s'inscrit dans les orientations» ensuite énumérées, sans distinction de genres argumentatifs et narratifs. Le «cadre argumentatif » est ainsi précisé :

- article (éditorial, article polémique, article critique, droit de réponse...);

- lettre (correspondance avec un destinataire défini dans le libellé du sujet, lettre destinée au courrier des lecteurs, lettre ouverte, lettre fictive d'un des personnages présents dans un des textes du corpus, etc.);

- monologue délibératif; dialogue (y compris théâtral); discours devant une assemblée;

- récit à visée argumentative (fable, apologue...).

6o. Plus encore, ce développement se trouve en contradiction avec le paragraphe suivant intitulé «Note sur la dissertation et l'écriture d'invention» qui se termine par la considération suivante : «Aussi convient-il de faire percevoir aux élèves que l'une et l'autre formes d'écriture [dissertation et écriture d'invention] participent au même travail de construction d'une façon d'exprimer son point de vue, par une argumentation de conviction dans un cas, par une argumentation de persuasion ou une mise en images dans l'autre», Acc., p. 95. Alors que le paragraphe précédent semblait renvoyer dissertation et écriture d'invention à une même opération intellectuelle de formation du jugement, celui-ci les sépare en renvoyant, selon les catégories construites à propos des objets d'étude, la dissertation à la conviction fondée sur des arguments rationnels et l'écriture d'invention à la persuasion qui vise la séduction et les affects. Autre opposition tout aussi schématique et qui, comme la précédente, ne prend pas en compte les finalités des différents genres.

6r. Note de service n² 2006-I 99 du 4-I 2-2006, Bulletin officiel $\mathrm{n}^{\circ}$ 46, i4 décembre 2006. 
On constatera que l'essai, qui figurait dans la liste en 200I, a été retiré, en raison notamment de la difficulté à définir le genre et que n'y figurent pas les préfaces dont sont pourtant friands les concepteurs de sujets du baccalauréat. Si nous retenons cette liste de 2006 comme base de réflexion pour les genres argumentatifs, une autre difficulté surgit, soulignée notamment par Yves Maubant :

[...] ces genres de discours ne sont pas des genres forcément littéraires, ou surtout suffisamment classiques, c'est-à-dire faisant l'objet d'un apprentissage dans les classes. D'où pour les élèves une absence possible de référents, de représentations intellectuelles, culturelles mais aussi sociales, personnelles ou affectives de ces textes à écrire. [...] on fait écrire pour cet examen [le baccalauréat] des textes dont on n'a parfois pas enseigné la lecture, des textes dont les élèves n'ont aucune pratique culturelle, sauf les rares qui lisent les préfaces, Le Monde littéraire ou Le Monde tout court ${ }^{62} ![\ldots]$

De fait, si les monologues de théâtre, les situations de dialogue, la fable et le conte philosophique ont pu être rencontrés et étudiés par les élèves, ce n'est pas le cas de l'article ou plutôt des articles, l'article ne pouvant de surcroît être défini comme un genre. Quant à la lettre, si souvent utilisée dans les sujets, elle n'était au programme que de la série $L$ en première avec l'objet d'étude consacré à «L'épistolaire» et elle a été sacrifiée à la réintroduction du roman lors de la récente modification des programmes ${ }^{63}$.

Sans entrer ici dans une discussion sur la littérarité, posons que les articles de presse, de manière générale, n'ont pas vocation à entrer dans le corpus des textes littéraires et que si certains peuvent témoigner de réelles qualités d'écriture, les préoccupations de leurs auteurs ne sont pas d'ordre stylistique. On peut penser que si des articles sont lus en classe, ils le sont sans doute pour leur contribution à un débat d'idées et non pour étudier un genre argumentatif particulier. Je retiendrai donc, pour la réflexion qui va suivre, la lettre, le dialogue, le discours devant une assemblée et le récit à visée argumentative (ou apologue). J'écarte le «monologue délibératif » pour des raisons qui tiennent à ce que j’ai dit plus haut à propos de la délibération : la délibération intime (pour reprendre les termes de Perelman) ne me parait pas être prioritaire pour l'apprentissage de l'argumentation dans la mesure où elle ne vise pas un destinataire autre que soi.

62. "Ambiguïtés de l'écriture d'invention au lycée: un champ non stabilisé», Pratiques, $\mathrm{n}^{\circ}$ I 27 -I 28 , décembre 2005 , p. 173.

63. Arrêté du 5-10-2006, Bulletin officiel $\mathrm{n}^{\circ} 40$ du 2 novembre 2006. 


\section{B. Pour le choix de genres aux finalités argumentatives claires et en prise sur des questions autres que littéraires}

Récapitulons tout d'abord les genres effectivement demandés dans les sujets d'invention argumentatifs de l'EAF :

\begin{tabular}{|l|c|l|c|}
\hline $\begin{array}{l}\text { Genres demandés dans } \\
\text { les sujets argumentatifs } \\
\text { métatextuels }\end{array}$ & $\begin{array}{l}\text { Genres demandés dans les } \\
\text { sujets argumentatifs non } \\
\text { métatextuels }\end{array}$ & \\
\hline Lettre & I 5 & Lettre $^{64}$ & 9 \\
\hline Dialogue & 9 & Dialogue $^{65}$ & 6 \\
\hline Article & 6 & Discours & 3 \\
\hline Préface & 3 & Apologue & 2 \\
\hline $\begin{array}{l}\text { Texte de présentation d'un autre } \\
\text { texte }\end{array}$ & 2 & Monologue délibératif & I \\
\hline Discours & I & Article (Éloge) & I \\
\hline
\end{tabular}

Dans le cas des sujets métatextuels, la lettre est le genre le plus représenté, suivi du dialogue et de l'article. Bertrand Daunay s'est interrogé sur les liens entre écriture d'invention et écriture métatextuelle et a recherché les antécédents de cette confusion à travers le cas particulier de la lettre. Il a ainsi montré que dans les années I930-I940, la lettre était sortie progressivement de son origine générique (qui en justifiait la présence dans l'enseignement rhétorique) pour intégrer l'écriture métatextuelle qui domine de plus en plus l'enseignement du français ${ }^{66}$.

Ce qui explique la facilité avec laquelle la lettre a pu être ainsi utilisée pour le discours critique, c'est qu'il s'agit, rappelons-le, d'un genre à la forme très libre. On pourrait d'ailleurs dire la même chose du dialogue, autre genre très utilisé dans l'écriture d'invention. En dehors d'une situation de communication qui précise pour la lettre le locuteur et le destinataire et, pour le dialogue, le locuteur et l'allocutaire, les contraintes formelles sont presque inexistantes. Il s'agit de ce j'ai appelé des genres «non marqués» ou par défaut, de même que l'article et la préface. Ce caractère prédispose donc ces genres à se trouver attirés dans l'orbite de l'écriture critique et, en particulier, de celle des genres scolaires de la dissertation et du commentaire qui, en l'absence de modèles génériques clairement identifiés, jouent le rôle de modèles

64. Dont une lettre narrative à valeur d'apologue.

65. Dont trois dialogues de théâtre et un dialogue dans un apologue.

66. "Les liens entre écriture d'invention et écriture métatextuelle dans l'histoire de la discipline : quelques interrogations», Enjeux, $\mathrm{n}^{\circ}$ 57, septembre 2003. 
scolaires et les orientent vers une écriture argumentative à finalité littéraire ou esthétique.

Mais si la lettre peut permettre de tenir un discours critique sur un texte ou de réfléchir sur la littérature, elle peut aussi accueillir toutes les formes de discours. Certains sujets d'ailleurs l'utilisent pour demander une narration (Mme de Grignan à sa mère, la tante de George Sand à sa nièce), avec changement de registre et de style ou de point de vue. La lettre n'est donc pas prédestinée à l'écriture critique et il n'est pas étonnant qu'on la retrouve aussi en tête des sujets argumentatifs non métatextuels, quoique de manière moins massive, suivie également du dialogue. Par commodité, j’ai parlé jusqu'ici de «genre» pour le dialogue, mais les sujets d'invention du baccalauréat qui demandent un dialogue ne se réfèrent pas tous au dialogue comme genre littéraire autonome puisque certains sont des dialogues de théâtre ou s'inscrivent dans un genre narratif (pour les sujets argumentatifs non métatextuels). Un seul sujet s'appuie sur un modèle de dialogue présent dans les textes du corpus, en l'occurrence un extrait du Neven de Ramean de Diderot pour un sujet de 2006 (série L, centres étrangers). Les autres demandent d'inventer des dialogues dont le seul modèle implicite semble être celui de la conversation courante et non des modèles littéraires de référence. Le dialogue est alors simplement une forme commode d'échange d'idées, considérée comme moins difficile que la dissertation.

Les deux genres argumentatifs les plus contraignants, le discours et l'apologue, sont les moins représentés. On s'en étonnera pour l'apologue, qui est pourtant un genre privilégié par les programmes et accompagnements de première, et qui est très représenté dans les corpus de l'EAF. Mais de l'étude de l'apologue à son écriture, il y a un pas que peu de sujets franchissent. Car l'apologue conjugue narration et argumentation, fiction et réflexion morale et/ou politique. Il est donc un genre dont l'apprentissage est forcément difficile et dont la production, dans le temps limité de l'épreuve écrite du baccalauréat, est également périlleuse.

Quant au discours devant une assemblée, on peut s'interroger sur le très faible nombre de ses occurrences dans les sujets d'invention, y compris lorsque le sujet de l'argumentation n'est pas métatextuel. En ce qui concerne les corpus, il y est tout aussi absent. Paradoxalement, alors que le discours, genre argumentatif par excellence, couronnait autrefois l'étude des humanités et était l'exercice privilégié de la classe de rhétorique, il peine à retrouver une place dans l'enseignement des lettres au moment où les programmes font une place importante à l'argumentation. Sans doute peut-on y lire, de la part des concepteurs des programmes, la crainte de donner l'impression d'un retour à une rhétorique pré-lansonienne. 
Il importe donc de donner une place mieux définie à ces trois genres que sont la lettre, le dialogue et le discours dans la perspective de travaux d'écriture d'invention. Ces genres relèvent en effet, contrairement à l'apologue, de ce que les programmes appellent l'argumentation «directe». Les choisir pour l'enseignement de l'argumentation revient donc à clarifier la situation d'argumentation et ses enjeux. Leur intérêt didactique est en effet de présenter un destinataire identifié, une situation de communication fictive mais définie et un enjeu argumentatif clair. Caroline Golder et Delphine Pouit, qui travaillent sur l'argumentation en psychologie cognitive, insistent, dans la conclusion de leur article «Quelles situations pour apprendre à argumen$\operatorname{ter}^{67}$ ?», sur l'importance de la finalité communicative des textes argumentatifs produits. Elles opposent à la dissertation qui «consiste peut-être essentiellement à construire réflexivement sa position, et non à défendre sa position» et dont l'enjeu réel est «de produire un discours argumentatif dont la finalité n'est pas de convaincre que l'on a raison, mais de convaincre que l'on sait argumenter», un discours argumentatif élaboré «dans lequel un locuteur, parce qu'il se sent engagé en tant qu'acteur social, va défendre sa position par des arguments qu'il considère comme les plus convaincants tout en faisant une place aux contre-discours qu'il sait se tenir sur la question débattue». La caractéristique essentielle d'un discours argumentatif étant qu'il vise à agir sur un destinataire donné, il importe donc que ce destinataire soit non seulement précisé (et c'est ce que font déjà les sujets d'invention du baccalauréat) mais que les élèves puissent avoir une représentation de ce destinataire (et il est moins sûr que ces mêmes sujets le permettent). Comme l'écrivent Caroline Golder et Monik Favart à propos du destinataire de l'argumentation dans un autre article : «son degré de familiarité avec le locuteur apparait comme une variable déterminante des types d'argument fournis et du degré d'élaboration textuelle des discours tenus ${ }^{68} \gg$. S'interrogeant sur l'identification de ce destinataire dans l'exercice scolaire de l'argumentation écrite, elles poursuivent :

Les élèves argumentent-ils réellement ou fabulent-ils sur ce qu'ils croient être attendu par l'enseignant sur une question donnée (argumenter en proposant des «lieux communs» pour ne pas «prendre de risques» tout en montrant que l'on sait argumenter)? À qui est destinée l'argumentation? À tout le monde (l'auditoire est universel et, dans ce cas, l'exposition de «lieux communs» constitue effectivement une stratégie prudente), à l'enseignant (l'identification, par l'élève, de ses positions sur la question paraît alors primordiale), ou encore à un interlocuteur «fantôme»? Les contraintes rhétoriques de l'argumentation semblent alors dans cette situation encore plus difficiles à prendre en compte. Pourtant lorsque les

67. Le Français aujourd'bui, n' ${ }^{\circ} 2_{3}$, septembre 1998.

68. C. Golder et M. Favart, «Argumenter c'est difficile... oui, mais pourquoi? Approche psycholinguistique de la production argumentative en situation écrite», Études de linguistique appliquée, $\mathrm{n}^{\circ} \mathrm{1} 30$, avril-juin 2003, p. $20 \mathrm{I}$. 
élèves sont «guidés» dans des situations dans lesquelles on les incite à envisager et à évaluer les arguments qui soutiennent un point de vue adverse (par exemple, en se mettant à la place de quelqu'un qui aurait une position contraire à la leur), ils améliorent nettement leurs performances initiales en produisant davantage de contre-arguments et de raisons pour soutenir ces contre-arguments. (p. 20I)

L'autre paramètre situationnel déterminant identifié par les psycholinguistes est celui de la nature du thème à débattre. Lorsque le thème est familier en effet, "quel que soit l'âge des élèves, les textes contre-argumentatifs dans lesquels le rédacteur tient compte des différentes positions possibles sur un thème $[\ldots]$ sont plus fréquents» (p. ${ }^{202^{69}}$ ). Les auteurs précisent ce qu'elles entendent par «thème familier» :

Le thème leur est familier non seulement parce qu'ils connaissent les différents arguments qui peuvent être avancés à son sujet, mais aussi parce qu'ils se sentent véritablement impliqués dans le débat. La position qu'ils défendent n'est pas seulement un point de vue théorique, elle peut avoir un certain nombre de répercussions pratiques dans leur vie quotidienne [...]. (loc. cit.)

La lettre, le dialogue et le discours peuvent justement tout à la fois proposer des situations de communication dont le destinataire ait un certain degré de familiarité avec les élèves et dont le thème les intéresse. J'entends ici par «degré de familiarité» avec le destinataire non pas, s'agissant du lycée, des membres de leur entourage proche (parents, copains par exemple) mais tout destinataire dont ils puissent avoir une certaine représentation. J'exclus donc par exemple «la lettre à votre éditeur», l'article pour une «revue littéraire» ou une «revue de poésie», ou même la lettre à l'ami fictif «à qui on fait part de ses réactions face à la lettre de Mme du Deffand» ou à ce bien étrange ami «qui vous écrit que la littérature n'a pas pour but d'apprendre à réfléchir mais de distraire et d'émouvoir», sans parler de ce dialogue tout aussi artificiel «entre un interlocuteur qui pense que la poésie permet d'exprimer une expérience personnelle et un autre qui, au contraire, la critique en l'accusant d'être un travestissement, voire une trahison de cette expérience». En revanche, on peut très bien concevoir une situation de communication fictive, tirée des textes du corpus, et dont les éléments de représentation sont précisément donnés par ces textes. Ce sera le cas, par exemple, lorsqu'on demande aux élèves d'imaginer un dialogue théâtral entre des personnages (à condition, bien sûr, que les textes du corpus donnent suffisamment d'éléments pour permettre de reconstituer une situation d'argumentation et ses enjeux ${ }^{70}$ ).

69. Voir également C. Golder, Le Développement des discours argumentatifs, Neuchâtel, Delachaux et Niestlé, I 996, et l'article déjà cité de C. Golder et D. Pouit.

70. Par exemple: «Dans l'extrait de La guerre de Troie n'aura pas lieu, Andromaque expose le point de vue des femmes et les raisons pour lesquelles elles condamnent la guerre. Écrivez un dialogue théâtral dans lequel Hector, l'époux d'Andromaque, expose le point de vue des 
Quant aux enjeux argumentatifs des sujets d'invention, ils peuvent recouvrir des questions de toute sorte : politiques, morales, sociales, esthétiques aussi bien sûr, mais dans ce dernier cas, en veillant à ce que ces questions ne soient pas hors de portée des élèves et qu'elles ne soient pas uniquement cantonnées à des réflexions métapoétiques qui les ramènent à la dissertation (dont le sujet d'invention se veut par ailleurs une solution alternative). Ainsi, demander à des élèves de rédiger un article polémique pour défendre ou attaquer les positions de Victor Hugo dans Réponse à un acte d'accusation, ou au contraire d'incarner Victor Hugo écrivant un article pour défendre le choix de tout écrivain de mettre la littérature au service de grandes causes, ne peut avoir d'enjeu argumentatif pour la majorité des élèves, de même encore qu'un discours défendant la poésie à l'occasion du «Printemps des poètes». En revanche, pour le sujet suivant donné en 2007 (série ES-S) : «À son arrivée à la Chambre des Pairs, le narrateur du texte $\mathrm{B}$, sous le coup de l'émotion, prend la parole à la tribune pour faire part de son indignation et plaider pour plus de justice sociale. Vous rédigerez ce discours», l'enjeu argumentatif est clair. Mais les éléments de contextualisation (un extrait de Choses vues) restent maigres et s'il n'y a pas eu de véritable apprentissage du genre du discours pendant l'année, la transposition d'un genre (le témoignage) à un autre (le discours) est loin d'aller de soi. Le «corrigé» s'imposera, puisqu'on peut renvoyer ensuite les élèves au discours du 9 juillet I 849 de Victor Hugo dit «Discours sur la misère». Mais sans véritable connaissance du contexte historique et sans pratique du genre du discours, les élèves ne peuvent produire que des textes pleins de bons sentiments. Pour renforcer le degré de familiarité des élèves avec le sujet, on aurait pu imaginer de transposer le discours à l'époque contemporaine puisqu'il y a toujours lieu de "plaider pour plus de justice sociale». Mais il faut aussi avoir fait du discours oratoire - et plus particulièrement ici de l'éloquence délibérative - un objet d'apprentissage afin que les élèves l'aient déjà pratiqué et puissent en utiliser les ressources argumentatives de manière concertée, en ayant conscience du cadre institutionnel et des enjeux politiques du genre.

Un seul sujet (2004, série technologique, Polynésie) se risque à proposer un corpus entier de discours : trois relèvent du délibératif (Mirabeau, Danton, De Gaulle), un de l'épidictique (Malraux), chacun de ces discours

hommes et les raisons pour lesquelles lui aussi condamne la guerre. Il s'adresse à son père Priam en présence d'Andromaque... [Ces deux personnages interviendront nécessairement dans la scène théâtrale].» (2002, série S-ES). Mais le sujet peut difficilement être traité à partir du seul extrait de la pièce figurant dans le corpus. Même problème pour ce sujet de 2003 , série L : «Face à Antigone, Ismène sa sœur défend à son tour "les lois de la cité” (texte C). Écrivez le dialogue de type théâtral qui oppose les deux personnages.» 
ayant un enjeu fort : éviter la banqueroute, sauver la patrie, engager le destin de la France et appeler à la vigilance pour l'avenir. Mais le sujet d'invention affaiblit cet enjeu en proposant aux élèves d'endosser le rôle du «délégué du comité de vie lycéenne» de leur établissement qui a «l'occasion de [s']adresser à une centaine de [ses] camarades» et doit prononcer «un discours visant à convaincre [son] auditoire de la nécessaire participation de la jeunesse au devoir de mémoire». D’une part, la situation proposée est totalement artificielle, d'autre part, la formulation bien-pensante du thème du discours, qui engage les élèves à se faire les relais d'une injonction professorale et même nationale, ne peut guère susciter de motivation pour argumenter, malgré les consignes du sujet qui invitent à "présenter une progression argumentative cohérente» et à «recourir à des procédés rhétoriques expressifs et variés». Plus généralement, c'est plutôt dans les sujets pour les séries technologiques qu'on trouve les rares ouvertures de l'argumentation du côté de la vie politique et sociale (sans doute parce que ces élèves sont considérés comme $a$ priori moins «littéraires» que les autres). Mais ces sujets conjuguent tous une injonction bien-pensante peu stimulante et peu susceptible de donner lieu à une argumentation intéressante, et l'absence d'un modèle formel susceptible de guider les élèves :

Lily, un an après son installation à Paris, écrit à sa famille restée en Somalie. Elle dénonce l'intolérance et le racisme dont elle est la victime. Vous rédigerez cette lettre en tenant compte des situations évoquées dans le texte de Pierre Perret et en développant l'argumentation de Lily. (2005)

À supposer même qu'on puisse envisager la possibilité d'une telle lettre, son enjeu argumentatif est inexistant (une dénonciation pour quoi? Pour qui? On aurait pu imaginer au moins une lettre destinée à dissuader d'autres jeunes de se risquer à l'immigration, mais sans doute le sujet aurait-il été trop brûlant) et le genre de la lettre aux proches ne peut que conduire à une narration, non à une argumentation. Un autre sujet fournit une consigne encore plus «molle»:

On vient de vous faire part d'une injustice grave. Vous exprimez votre désapprobation dans une lettre argumentée, que vous adressez au courrier des lecteurs d'un journal. La situation d'injustice que vous aurez dénoncée dans votre lettre conduira à l'énoncé d'une morale. (2005, centres étrangers)

Les textes du corpus (un fabliau anonyme du XIII siècle, Le Prud'bomme qui sauva son compère, XIII ${ }^{\mathrm{e}}$ siècle; «L'Huître et les plaideurs» de la Fontaine, et un extrait du chapitre 6 de Zadig) relèvent de «l'argumentation indirecte», pour reprendre la terminologie des programmes, et devant la difficulté de faire écrire un apologue, les concepteurs du sujet ont choisi un thème vague et une situation de communication sans enjeu argumentatif. Un sujet plus récent 
recourt aussi à la lettre mais adressée cette fois à un «élu local», ce qui nous rapproche d'un enjeu politique réel :

Vous avez été témoin, dans votre propre commune, d'une scène proche de celle que décrit Rimbaud dans «Les Effarés». Vous la racontez dans une lettre à un élu local pour lui faire part de vos émotions et l'inciter à agir. (2007, ES-S)

Si l'on comprend bien le souci des concepteurs du sujet de partir d'une situation concrète pour faire argumenter sur la misère et non de la dénonciation générale que proposent les autres textes du corpus, ils méconnaissent le fait que «Les Effarés» n'est ni un témoignage, ni une chose vue, ni un cri d'indignation. Ils introduisent donc une relation de porte-à-faux entre l'énonciation supposée de l'élève et celle du poème. Du coup, ce qui se voulait une démarche inductive parait extrêmement factice et pour tout dire tiré par les cheveux. Quant au modèle générique, il est lui aussi inexistant, à moins qu'on considère que la lettre de Fénelon à Louis XIV, qui figure dans le corpus, puisse inspirer la lettre à un élu local du Xxi ${ }^{\mathrm{e}}$ siècle.

Que retenir de l'analyse de ces quelques exemples? Tout d'abord la nécessité de concevoir une didactique de l'écriture argumentative fondée sur l'apprentissage de quelques genres argumentatifs bien circonscrits et appuyés sur des modèles de référence. On proposera une double progression qui va du dialogue au discours d'une part, de la lettre privée à la lettre ouverte d'autre part, dont le modèle est très proche de celui du discours oratoire. On sait en effet que traiter la dimension dialogique de l'argumentation à l'intérieur d'une activité d'écriture monologique est encore difficile pour les lycéens ${ }^{71}$. En situation de dialogue oral, chacun peut prendre appui sur l'argument ou la réfutation de l'interlocuteur. À l'écrit, les positions antagonistes ou simplement différentes sont incarnées par deux locuteurs différents, ce qui facilite la décentration par rapport à ses propres opinions. Du dialogue oral au dialogue écrit, puis du dialogue écrit au discours monogéré, écrit mais aussi oral, avec prise en compte du destinataire, on voit s'esquisser une progression qui peut s'appuyer sur des modèles ${ }^{72}$. En ce qui concerne la lettre, on part, comme pour le dialogue, d'un genre formellement peu contraignant, mais qui impose de prendre fortement en compte le destinataire. Toute lettre particulière n'ayant pas une visée argumentative, il importe de recenser un certain nombre de cas ou de situations sur lesquelles travailler et rechercher

7I. Voir C. Golder et D. Pouit, «Quelles situations pour apprendre à argumenter?», art. cité.

72. On pourra ainsi se reporter, dans le même numéro, à la séquence proposée par J. Fay qui part de l'analyse des arguments échangés dans des passages de La Controverse de Valladolid de J.-Cl. Carrère pour aller vers la production d'un plaidoyer, sous une forme écrite et orale. 
des exemples de lettres intéressantes à analyser. On rencontre, par la même occasion, un certain nombre de pratiques sociales de référence (dans quels cas peut-on écrire à un «élu local»? et d'ailleurs, que recouvre concrètement la notion d'«élu local»?) dont le cours de français ne devrait pas se désintéresser. Le continum qui fait passer de la lettre privée à la lettre particulière, mais avec un enjeu public, et enfin à la lettre ouverte permet d'étudier et de pratiquer différentes situations d'argumentation. La lettre ouverte, telle qu'on en trouve des exemples notamment chez Zola dans son recueil La Vérité en marche, emprunte en grande partie son modèle générique au discours oratoire et il pourra être intéressant de comparer ces deux modes d'action par la parole dans un même contexte politique.

En ce qui concerne ensuite les sujets d'argumentation, il faudrait orienter davantage le sujet d'invention vers des réflexions qui concernent la vie de la cité. Alors que les programmes posent la formation du citoyen comme une des finalités de l'enseignement du français, au lycée comme déjà au collège, on ne peut que regretter une forme de pusillanimité dans les questions abordées à travers les sujets d'invention argumentatifs. L'image du «citoyen» qui se dessine à travers l'analyse de ces sujets est majoritairement celle d'un homme (prédominance du masculin oblige) occupé à défendre la poésie, à réfléchir sur l'intérêt et les limites de l'autobiographie, à justifier des choix de mise en scène et qui, lorsqu'il ne discute pas de ses goûts en matière littéraire, veut bien défendre quelques injustices et plaider pour la tolérance. Programme peu exaltant, on en conviendra, pour des adolescents d'aujourd'hui, et peu à même de les aider à comprendre le monde contemporain. Les sujets d'invention gagneraient donc à actualiser chaque fois que possible les questions développées dans les textes du corpus, de manière à montrer que la réflexion d'un auteur du passé peut aussi aider à éclairer le présent. Les corpus eux-mêmes gagneraient à recourir davantage à des formes d'argumentation «directe» : discours politiques, plaidoyers, lettres ouvertes, pamphlets, manifestes... et à considérer comme faisant partie pleinement du littéraire l'éloquence politique et judiciaire, et pas seulement l'éloquence épidictique. Pour cela d'ailleurs, il importe aussi de faire des choix théoriques qui permettent tout à la fois d'analyser ces textes et d'en écrire avec des outils adaptés. C'est pourquoi je plaiderai in fine pour un retour raisonné à une rhétorique bien comprise.

\section{Pour un retour raisonné à une rhétorique bien comprise}

Bien évidemment, il ne s'agit pas de promouvoir un retour à une rhétorique devenue à la fin du XIX ${ }^{\mathrm{e}}$ siècle, selon Lanson, «un art de parler bien sans penser», ou encore un enseignement qui «tendait à polir le goût et à 
développer l'attitude oratoire ${ }^{73} »$. Car si la rhétorique s'est ainsi vidée de sa substance, ce n'est pas en raison d'une restriction de la rhétorique aux figures, mais d'une fossilisation des matières de discours, empruntées le plus souvent à une histoire lointaine et sans lien avec les questions qui agitaient la société française de la deuxième moitié du XIX ${ }^{\mathrm{e}}$ siècle ${ }^{74}$. Alors que le XIX siècle commençant avait connu une restauration de la rhétorique, portée par un renouveau de la parole oratoire (et de son rôle en politique mais aussi dans le monde judiciaire ${ }^{75}$ ), le Second Empire a mis fin pour un temps à cette libération de la parole et contribué sans doute à isoler l'enseignement de la rhétorique et à le priver de tout écho dans la vie politique. La Troisième République aurait pu tenter de redonner vie à l'enseignement de la rhétorique, mais elle a préféré faire porter ses efforts sur la morale laïque de l'école primaire et laisser à l'histoire littéraire promue par Lanson le soin de défendre cette morale nouvelle qui devait rassembler les jeunes Français dans le culte de ses grands hommes et en particulier de ses grands écrivains :

L'histoire littéraire est un moyen de rapprochement entre des compatriotes que tout le reste sépare et oppose et c'est pourquoi j'oserai dire que nous ne travaillons pas seulement pour l'érudition, ni pour l'humanité, nous travaillons pour nos patries ${ }^{76}$.

Une tentative comme celle de Joseph Reinach qui publie chez Delagrave en I 894 Le «conciones» français. L'Éloquence Française depuis la Révolution jusqu'à nos jours. textes de Lecture, d'Explication et d'Analyse pour la classe de première, restera isolée dans sa volonté d'actualisation des modèles rhétoriques qu'il tire, pour la plus grande partie, de l'éloquence politique de Mirabeau jusqu'à Jules Ferry.

Pourquoi alors revenir à la rhétorique? Rappelons que si historiquement la rhétorique a été d'abord une tekhnê, une méthode raisonnée pour apprendre à

73. Cité par R. Fayolle, «Bilan de Lanson», dans L'Histoire littéraire aujourd'bui, H. Béhar et R. Fayolle dir., Armand Colin, I990, p. I4.

74. Sans refaire ici une histoire de l'enseignement de la rhétorique au XIX siècle, on rappellera les pages savoureuses que Jules Vallès consacre aux humanités dans L'Enfant : le narrateur y confie son incapacité à se mettre à la place de Thémistocle (pour traiter le sujet «Thémistocle haranguant les Grecs») ou des autres personnages qu'il doit faire parler : «jai quatorze ans, je ne sais pas ce qu'il faut faire dire à Annibal, à Caracalla, ni à Torquatus non plus!». Non seulement il ne peut «[se] figurer qu' [il est] un Latin», mais il vit douloureusement ce qu'il ressent comme un exercice d'hypocrisie et de mensonge : «Je dis dans ces narrations qu'il n'y a rien comme la patrie et la liberté pour élever l'âme. Je ne sais pas ce que c'est que la liberté, moi, ni ce que c'est que la patrie. [...] Je me moque de la Grèce et de l'Italie, du Tibre et de l'Eurotas.» (L'Enfant, Gallimard, «Folio», 2000, p. 288 à 292).

75. Voir la contribution déjà citée de Fr. Douay et ma thèse : L'Éloquence parlementaire sous la monarchie de Juillet. Guizot, Thiers, Tocqueville, Grenoble 3, 2000.

76. «L'esprit scientifique et la méthode de l'histoire littéraire», conférence de novembre I909, citée par R. Fayolle, op. cit., p. I4. 
composer des discours, elle a pu permettre aussi très tôt d'analyser des œuvres. La plus grande partie des écrivains jusqu'au XIX ${ }^{e}$ siècle et même au-delà ayant été formée par la rhétorique, il n'est pas inutile pour nous non plus de savoir ce que les uns ou les autres lui doivent. C'est ainsi que la redécouverte de la rhétorique a pu apporter d'autres éclairages à la lecture du théâtre classique ${ }^{77}$. En ce qui concerne plus précisément l'argumentation, la rhétorique est un instrument d'analyse et de production privilégié parce qu'elle a été conçue à partir de - et pour - des situations de parole publique à visée explicitement argumentative : elle s'attache aux projets argumentatifs avoués, à la différence de la pragmatique (pour laquelle l'argumentation fait partie du sens) ou de la position qui considère que l'argumentation traverse l'ensemble des discours. La rhétorique permet donc d'abord et avant tout l'analyse et la production des discours, entendus comme genre de parole publique s'adressant à un auditoire déterminé. Ainsi les plaidoiries, les discours parlementaires, les harangues, les sermons, ou les éloges funèbres. Mais elle ne s'y limite pas : toute parole, écrite ou orale, ayant un projet explicite d'influence sur un auditoire peut justifier d'une analyse rhétorique. Ainsi, et de toute évidence, une lettre cherchant à convaincre son destinataire d'agir dans un sens déterminé et a fortiori une lettre publique qui cherche à modifier l'opinion d'un large auditoire relèvent pleinement d'une analyse rhétorique et peuvent être écrites en se fondant sur certaines des techniques de composition auxquelles a réfléchi la rhétorique.

Quelles notions pouvons-nous retenir de la rhétorique pour en faire un enseignement explicite? Quels outils donne-t-elle pour l'étude de l'argumentation étant entendu qu'il ne s'agit en aucun cas de se référer à une rhétorique normative ou prescriptive? Rappelons tout d'abord que la rhétorique vise une approche globale des discours (alors que la linguistique a tendance à s'emparer de certains concepts, comme l'ethos, et à les traiter indépendamment de l'ensemble du système). Ses parties essentielles sont l'inventio et la dispositio et non l'étude des figures, à quoi elle est encore trop souvent ramenée aujourd'hui. Elle considère le discours (au sens rhétorique du terme) comme un tout : en réception, elle étudie la structure d'ensemble du discours, son unité organique; en production, elle propose une technique de composition. Le choix des moyens verbaux dépend des stratégies d'ensemble, le discours étant conçu comme dynamique. En cela, la rhétorique est bien une grammaire

77. Voir par exemple G. Forestier, Introduction à l'analyse des textes classiques: éléments de rhétorique et de poétique, Nathan ("I28»), 1993, rééd. 2005; Fr. Goyet, «Les lieux de la pitié dans Athalie», Styles, genres, auteurs, n 3, 2003, p. 9I-I03; voir également dans G. Declercq et M. Rosselini dir., Jean Racine, I699-1999: actes du colloque, Ile-de-France - La Ferté-Milon, 2530 mai, PUF, 2003. 


\section{ÉCRITURE D'INVENTION ET ARGUMENTATION}

de texte et les figures de pensée (et non les figures de mots) constituent les briques les plus petites de sa construction. Elle s'enrichit en outre aujourd'hui de tout l'apport de la linguistique de l'énonciation qui permet une étude plus précise des moyens linguistiques mis en œuvre dans la stratégie persuasive. Technique, moyens: ces termes pourraient laisser craindre une approche formelle de l'argumentation. Mais il n'en est rien : née de l'expérience de la parole publique, la rhétorique est liée fondamentalement à la vie de la cité et l'ensemble des moyens sont mis au service des idées à défendre.

L'inventio propose une réflexion sur les preuves : celles qui relèvent des faits et du raisonnement, bien sûr, mais aussi celles qui concernent l'ethos et le pathos. Au lieu de disjoindre la raison et les passions, elle les pense en les articulant et permet d'aborder de manière beaucoup moins naïve et simpliste les questions de manipulation qu'il ne suffit pas de renvoyer du côté des affects. Vérité et vraisemblable, réel et fiction, schèmes de raisonnement, croyances partagées mais aussi valeurs au nom desquelles on parle, ce sont tout autant de notions auxquelles la rhétorique permet de réfléchir. La notion d'ethos (ou mœurs), caractère de l'orateur, tel qu'il paraît dans le discours mais aussi celle des mœurs des auditeurs, que l'orateur doit connaitre pour proportionner son discours à leur intelligence, leurs sentiments permettent de penser la nécessaire adaptation à l'auditoire. Quant au pathos, longtemps si mal compris et dressé contre la raison, il permet de réintégrer la question des émotions dans l'argumentation et d'apprendre à distinguer par exemple le recours aux émotions immédiates et passagères (comme l'apitoiement devant les victimes de telle catastrophe ou celles de tel tueur en série) et l'appel aux grands sentiments, qu'il s'agisse du salut de l'État, de la justice ou plus largement d'un attachement à une certaine idée de l'homme et de l'humanité. La dispositio permet de mettre en relation le choix des moyens pour convaincre et la construction du discours : retrouver les grandes parties du discours et les raisons qui ont présidé à son organisation est le premier moyen, à la lecture, de rentrer dans l'analyse de l'argumentation; et les conseils donnés pour la dispositio sont aussi un guide pour la composition du discours, y compris lorsqu'on décide de faire des choix différents de ceux qui sont habituellement recommandés. Enfin, la rhétorique bien comprise a un sens aigu de la forme qu'elle ne pense jamais indépendamment de la stratégie de persuasion qu'elle poursuit et donc des idées qu'elle défend. C'est pourquoi elle est également pleinement littéraire et ne doit pas se penser contre la littérature.

Il ne s'agit pas pour autant de revenir purement et simplement à la rhétorique d'Aristote, de Cicéron et de Quintilien en faisant fi de Perelman et de tous les apports de la linguistique de l'énonciation et de la pragmatique. Mais il faut repenser l'articulation de ces différentes théories, comme le fait notamment 
Ruth Amossy pour situer l'analyse argumentative du discours dans son introduction à L'Analyse dans le discours. Discours politique, littérature d'idées, fiction ${ }^{78}$. Il faut également faire le lien avec les sciences de la communication comme le propose Philippe Breton avec L'argumentation dans la communication ${ }^{79}$. Sa propre classification des arguments d'ailleurs, qui se veut «simple, rustique, en principe facile à mettre en œuvre dans l'analyse» (p. 42) pourrait être utilement proposée tant pour l'analyse que pour la pratique de l'argumentation au lycée. C'est donc en faisant de cette rhétorique, revue à la lumière d'autres théories qui la prolongent ou l'enrichissent, la théorie de référence pour l'analyse de l'argumentation et la production de genres argumentatifs comme le dialogue, la lettre et surtout le discours qu'on pourra redonner aux programmes la cohérence nécessaire pour clarifier l'apprentissage de l'argumentation. Ce recentrage sur la rhétorique demandera également un élargissement du corpus des textes à étudier vers des discours politiques et judiciaires notamment, élargissement déjà rendu possible par les programmes actuels mais en réalité peu pratiqué par les manuels et, nous l'avons vu, dans les sujets du baccalauréat.

Un tel programme ne peut que poser in fine la question de la formation des enseignants qui seraient chargés de le mettre en œuvre. Or, nous sommes actuellement loin du compte: pour la formation initiale, l'argumentation ne fait pas partie en tant que telle des études universitaires et les futurs enseignants ne la découvrent au mieux comme objet d'étude qu'en préparant l'épreuve sur dossier pour l'oral du CAPES. Certes, ils pratiquent dissertation et commentaire mais n'ont que rarement l'occasion de réfléchir de manière métadiscursive sur ce qu'est le discours argumentatif. Quant à la rhétorique, elle est sans doute mieux connue aujourd'hui, mais son enseignement est encore trop souvent conçu dans le cadre des enseignements de langue (influence des figures oblige), non dans celui de l'analyse des textes et encore moins pour l'apprentissage de l'écriture argumentative. La pratique de l'écriture d'invention est également peu répandue, sinon dans des options consacrées aux pratiques d'écriture ou dans des ateliers d'écriture qui ne sont pas malheureusement, faute de moyens, proposés à tous les étudiants. Enfin, si on constate un retour du discours oral dans les facultés de droit sous la forme de concours d'éloquence, les études littéraires restent en retrait, alors même qu'il existe aujourd'hui des concours d'éloquence pour les lycéens, comme celui du Mémorial de $\mathrm{Caen}^{8}$. Quant à la formation continue, réduite à la

78. Nathan Université, 2000. L’ouvrage a été réédité chez Armand Colin dans la collection «Cursus» en 2006.

79. La Découverte et Syros, 200 I ( ${ }^{\text {re }}$ éd. La Découverte, I996).

80. Ce concours existe depuis 1998 et invite les lycéens de moins de 20 ans à plaider pour les Droits de l'Homme. Des extraits de ces plaidoiries, ainsi que des vidéos des gagnants sont 
portion congrue, elle ne saurait, en l'état actuel, compenser les manques de la formation initiale. Une pratique raisonnée du discours argumentatif tant à l'oral qu'à l'écrit dans les études universitaires : vaste et noble programme sans doute, mais chantier nécessaire si on veut vraiment former, au-delà des étudiants de lettres, des générations de citoyens moins démunis.

consultables sur le site du Mémorial de Caen à l'adresse suivante : http://www.memorialcaen.fr/portail/index.php, rubrique «Concours». 